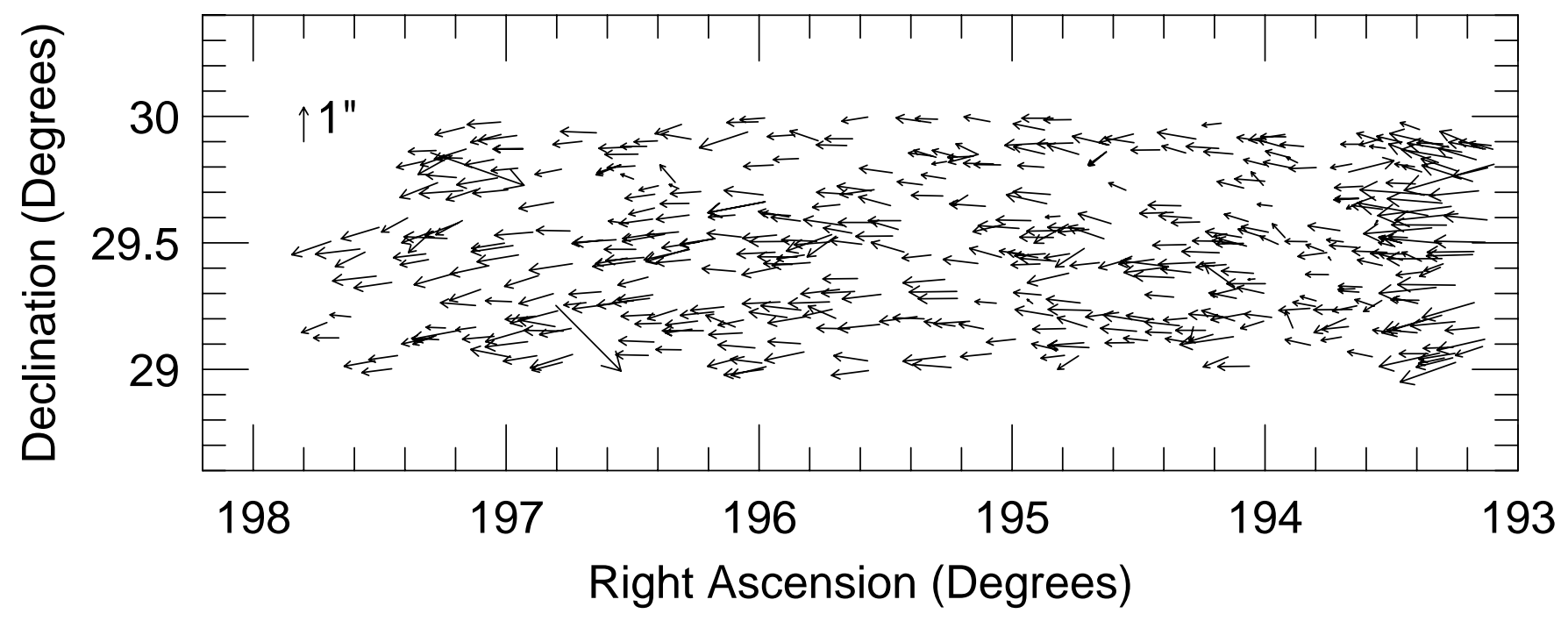




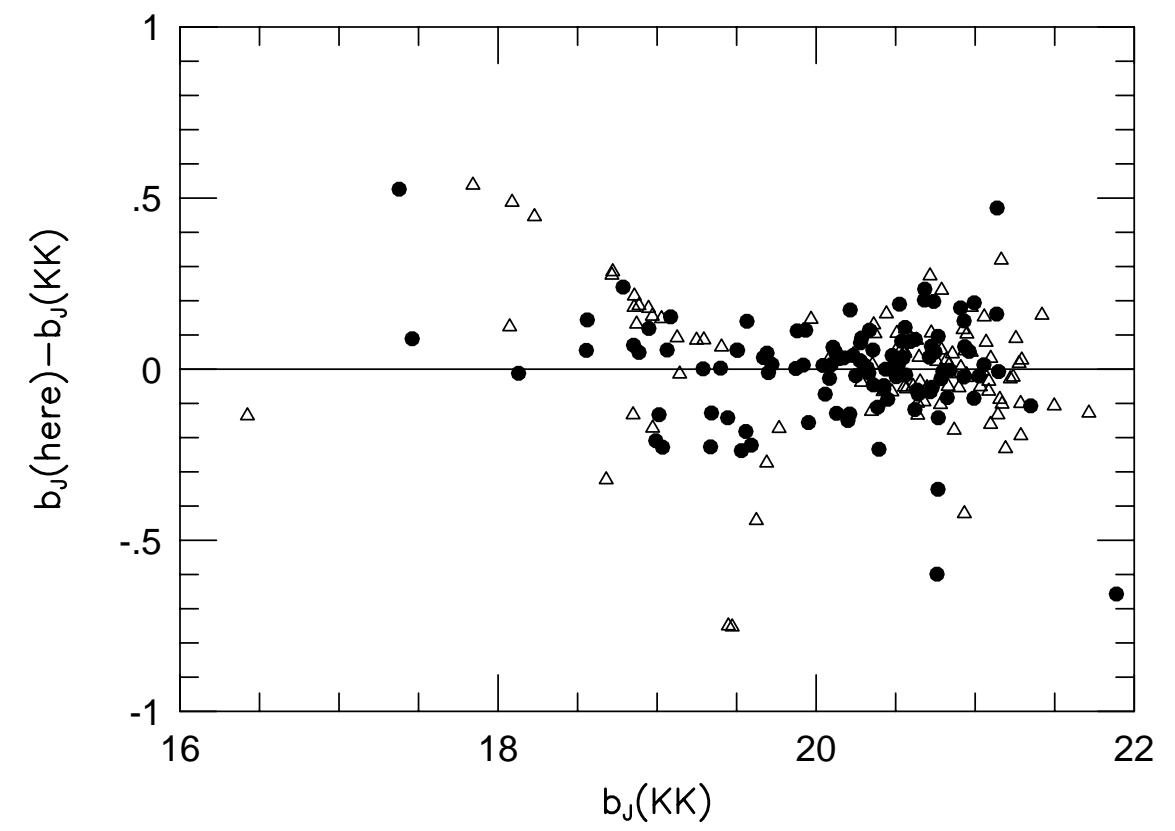




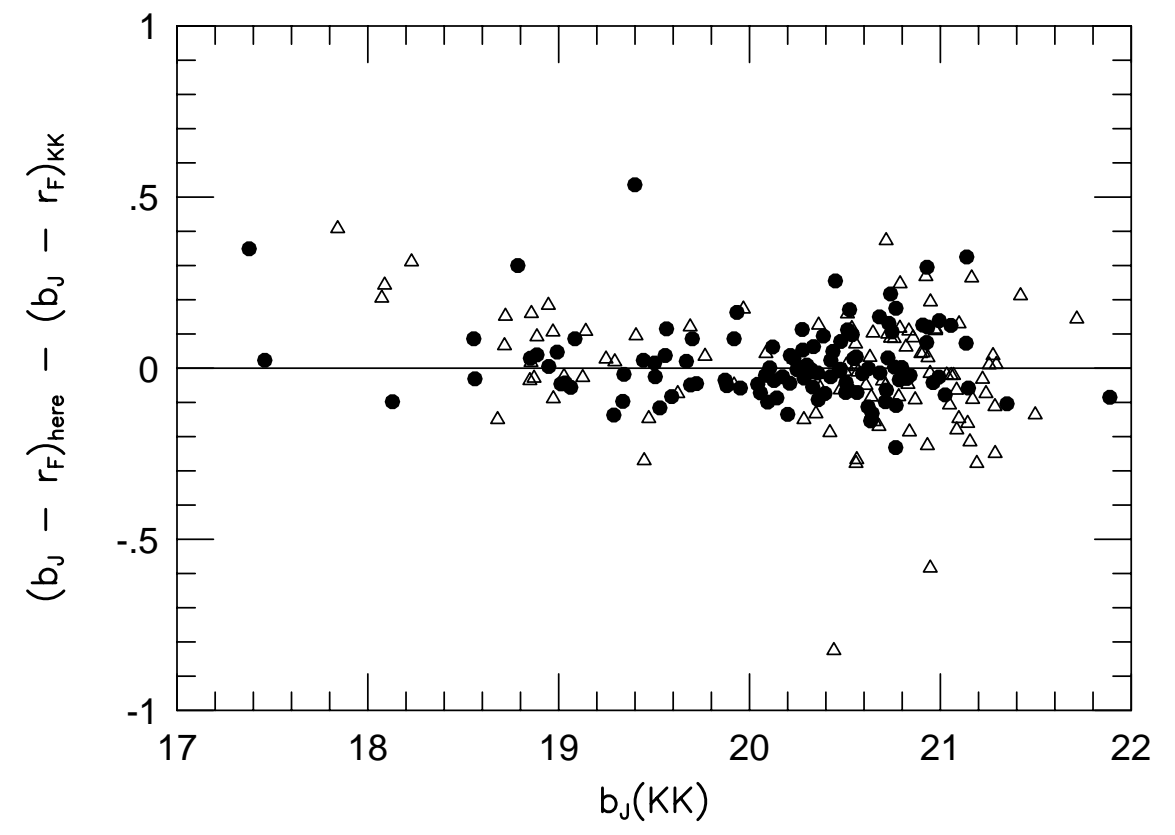




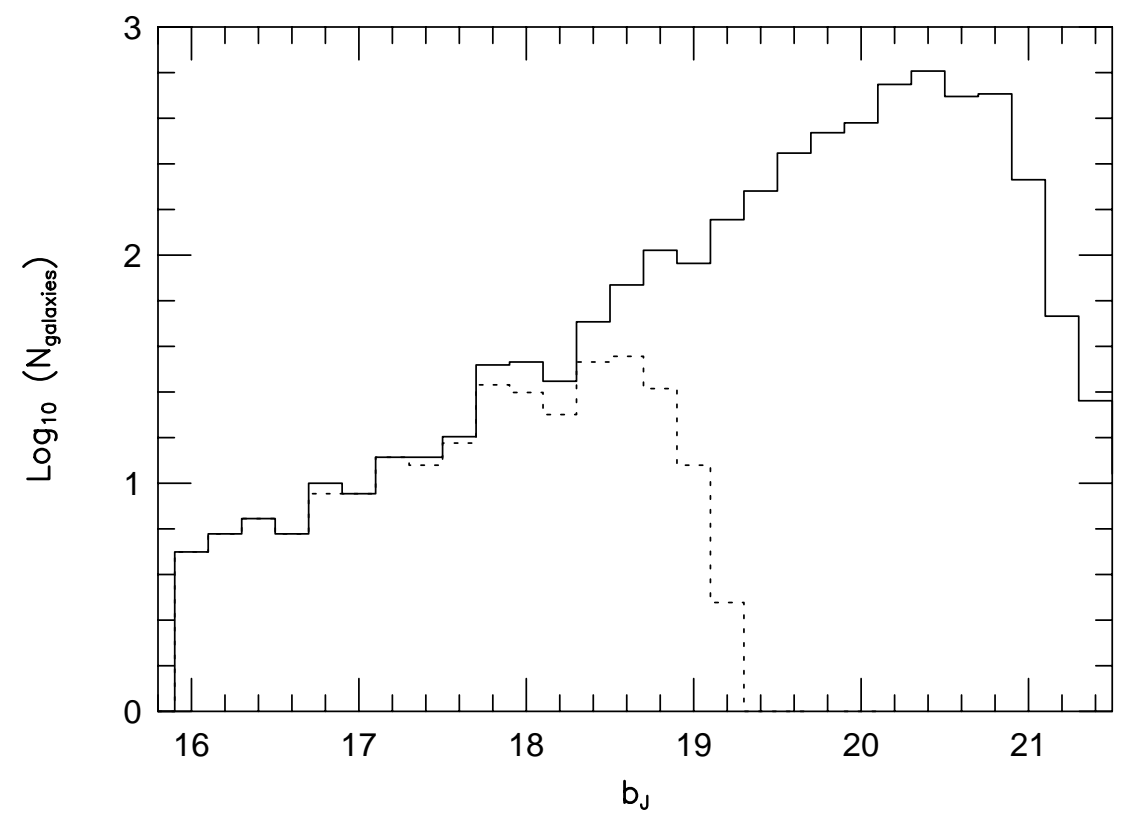




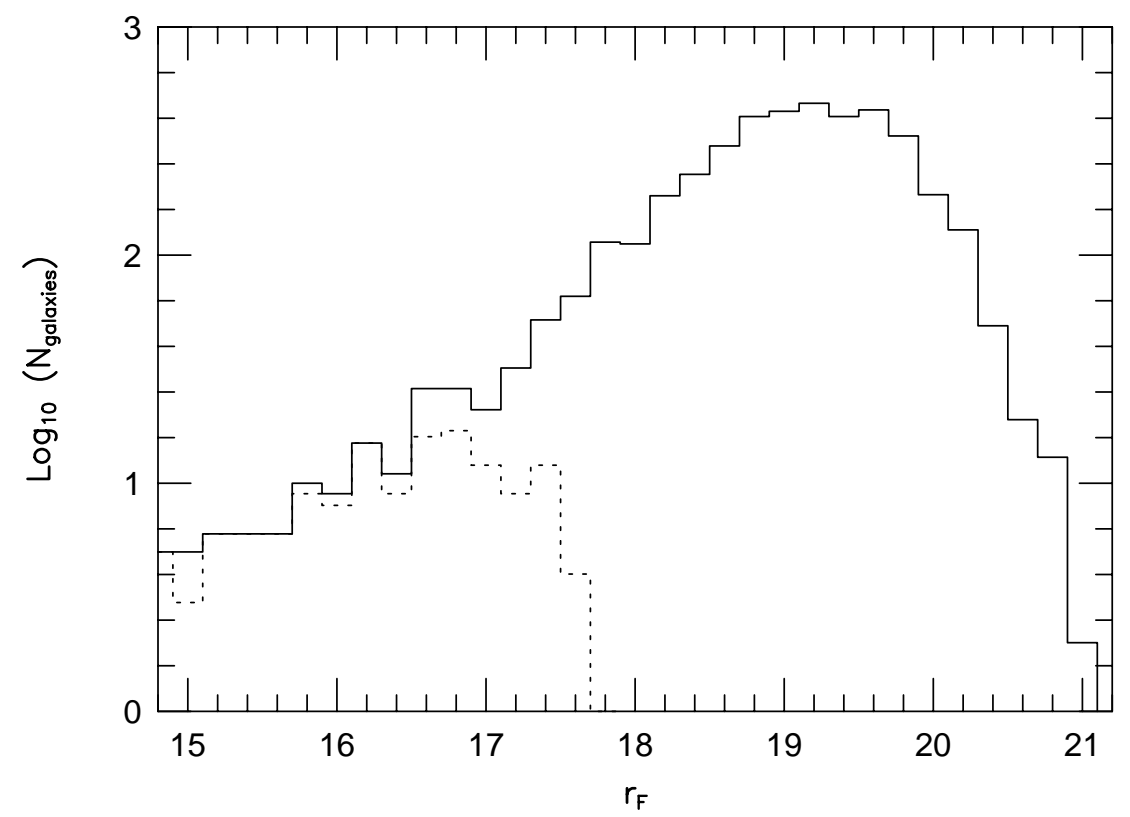




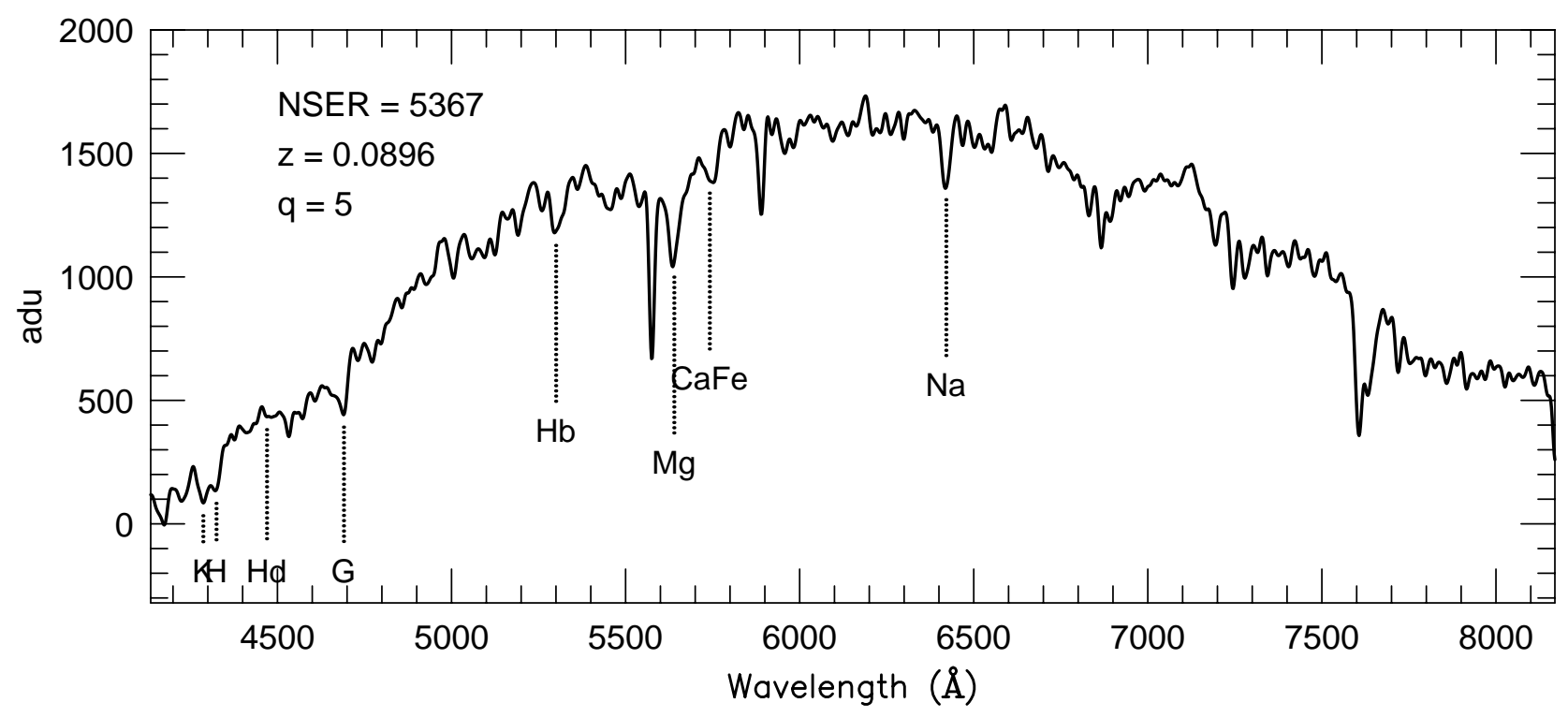




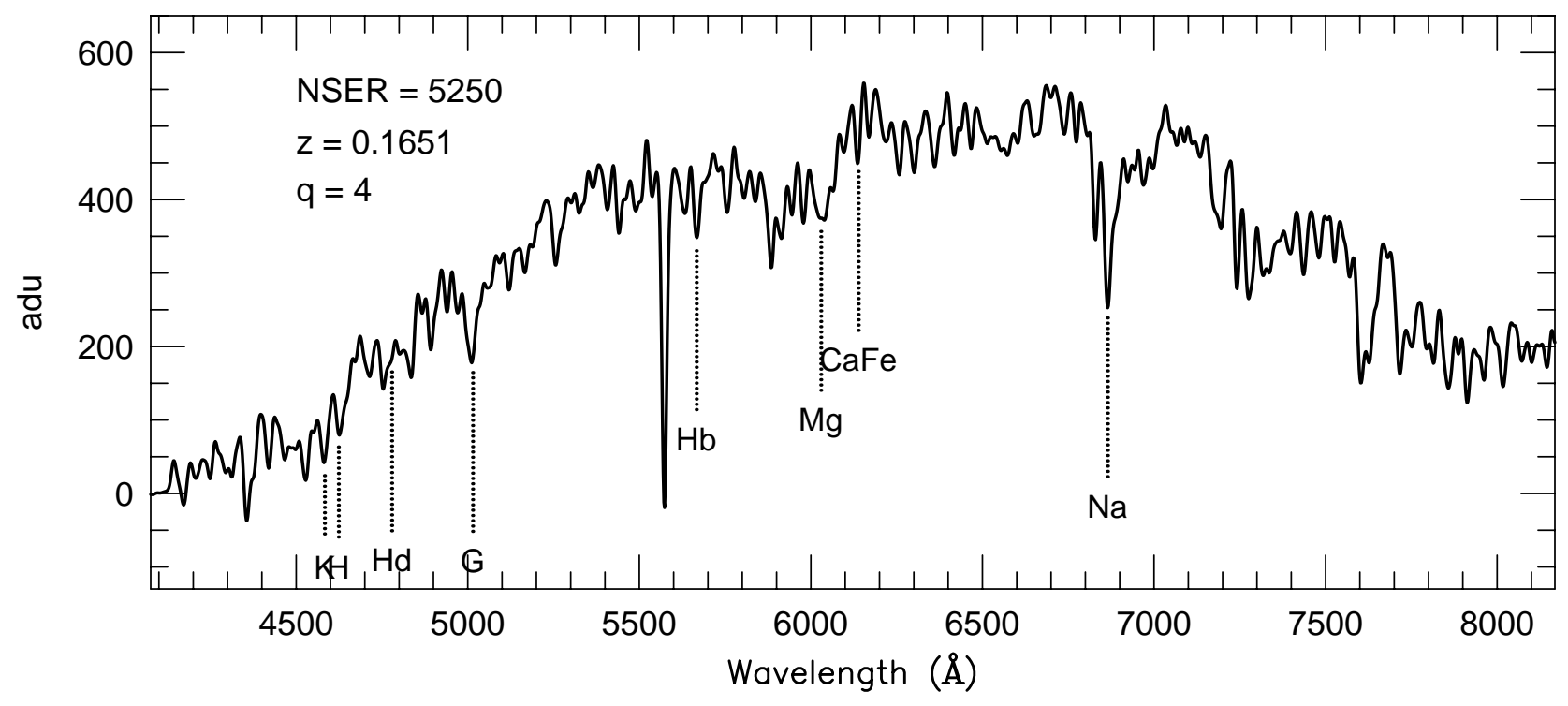




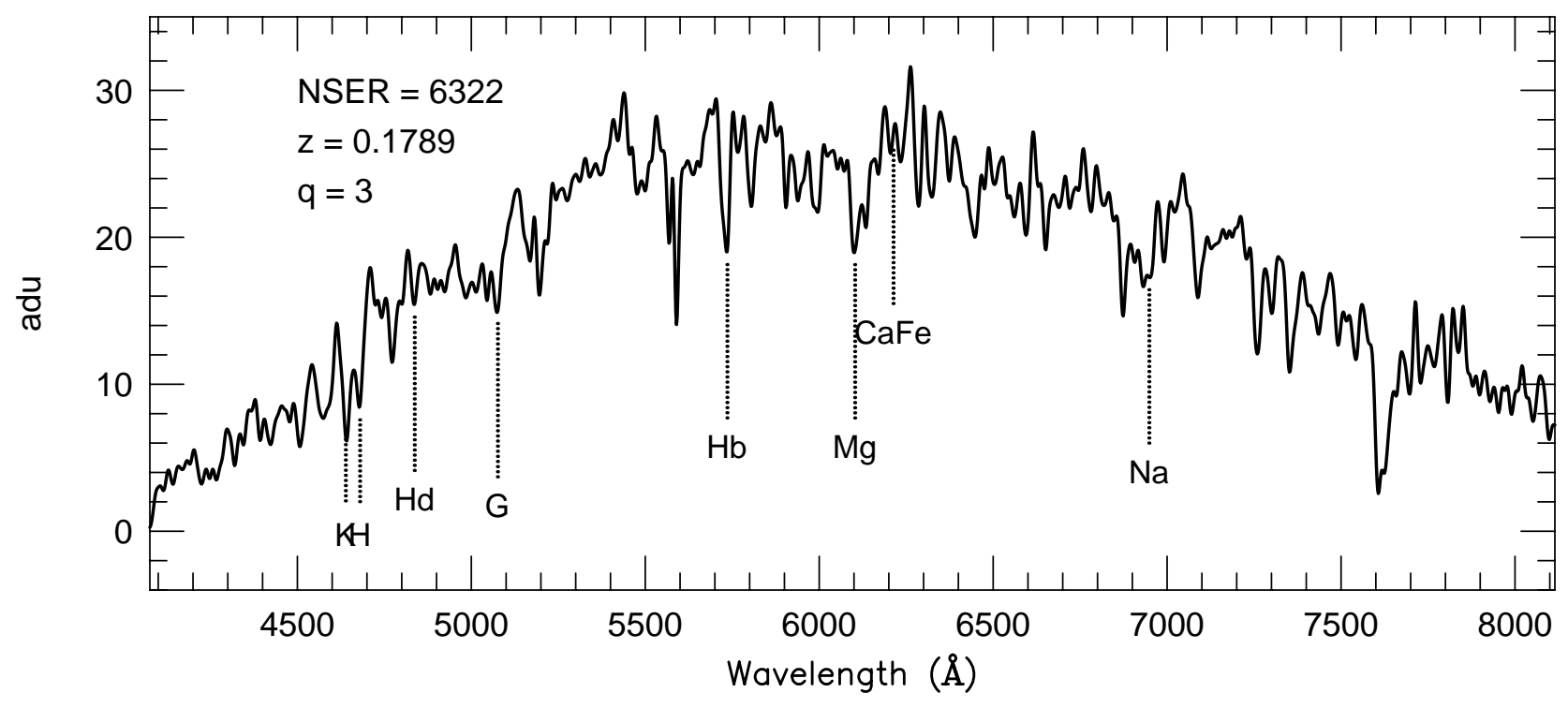




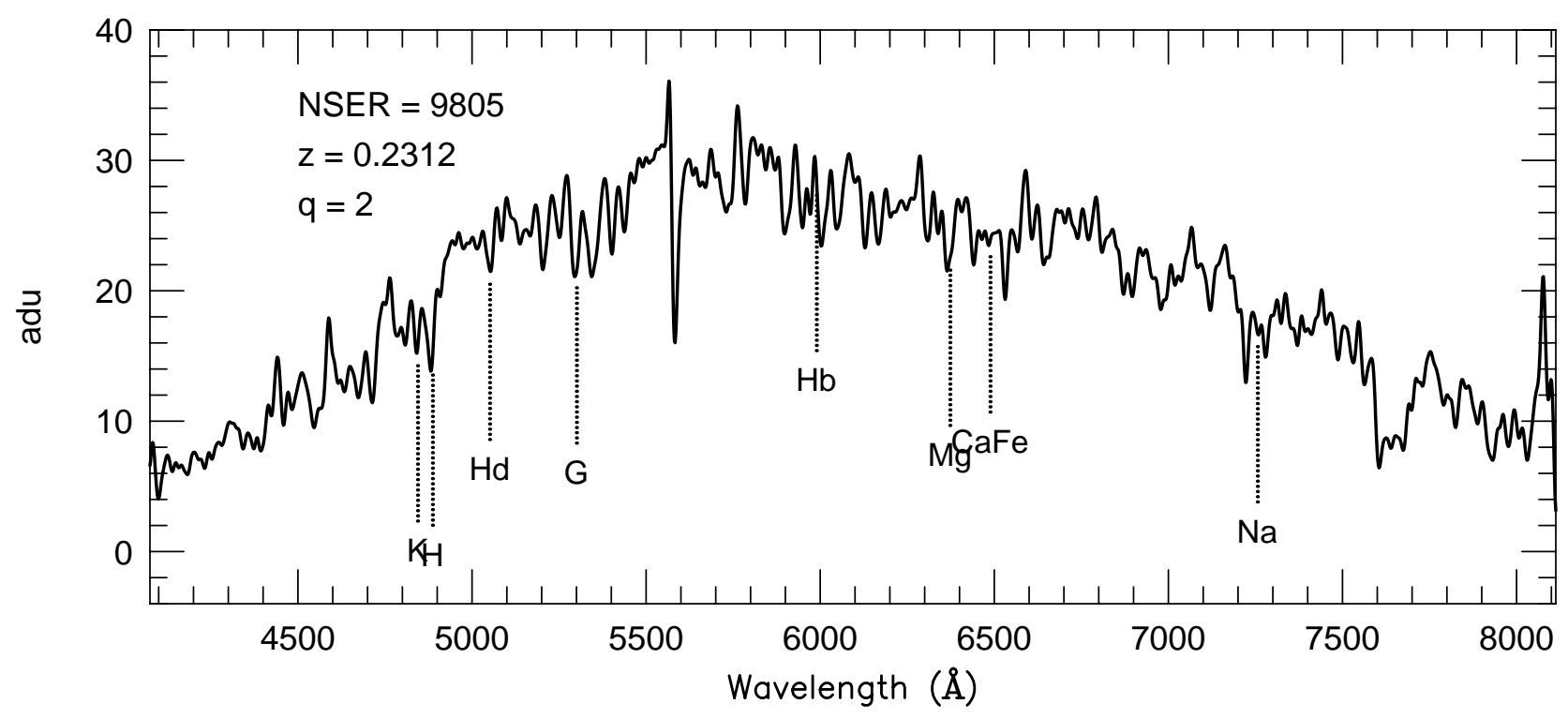




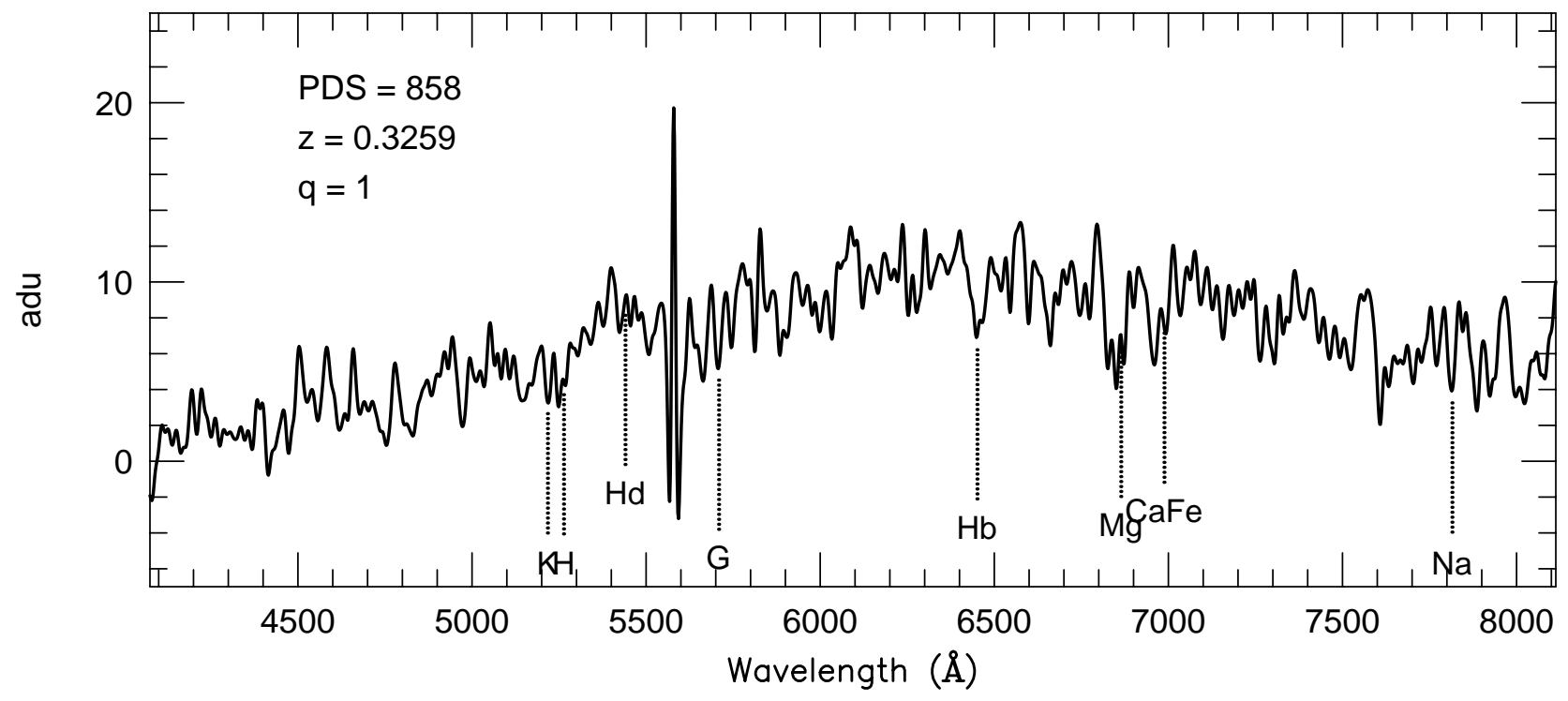




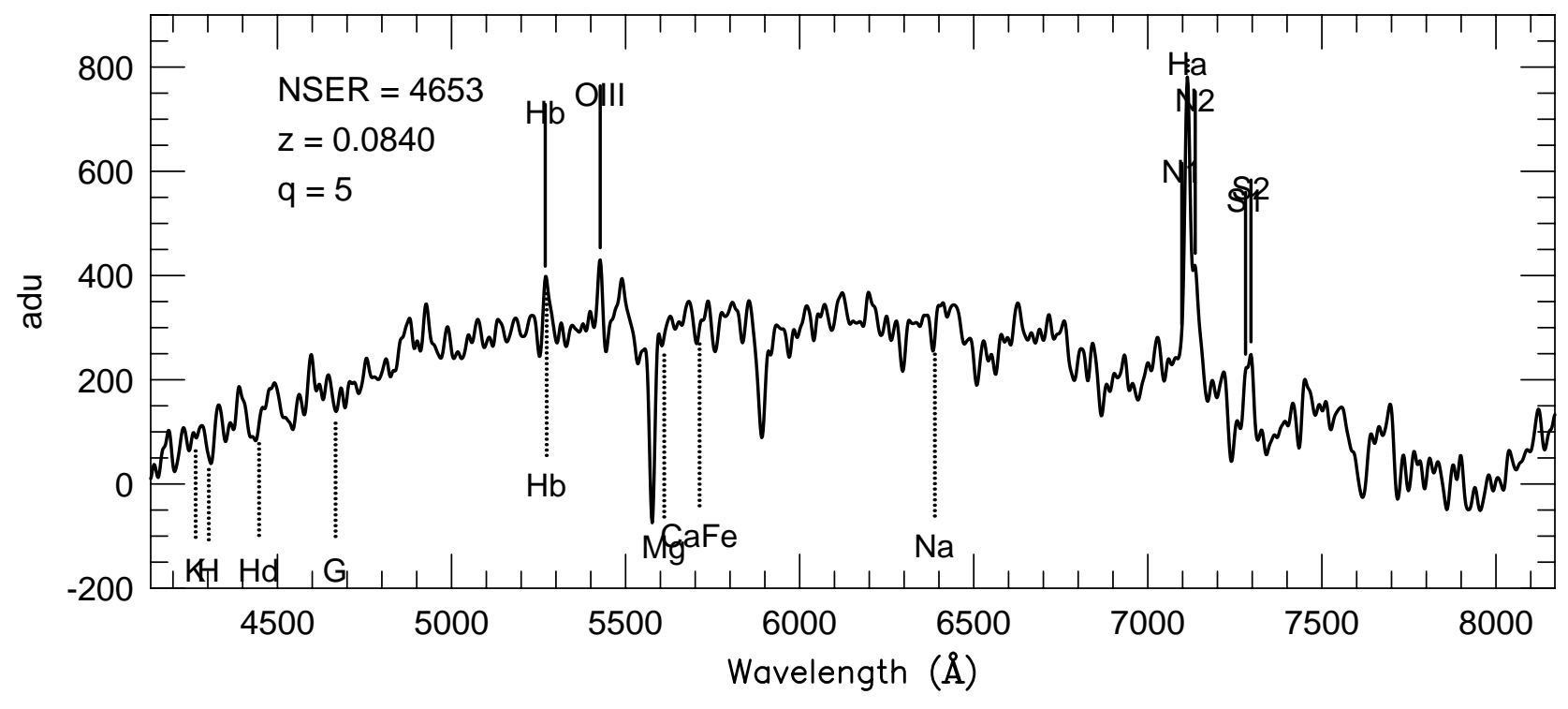




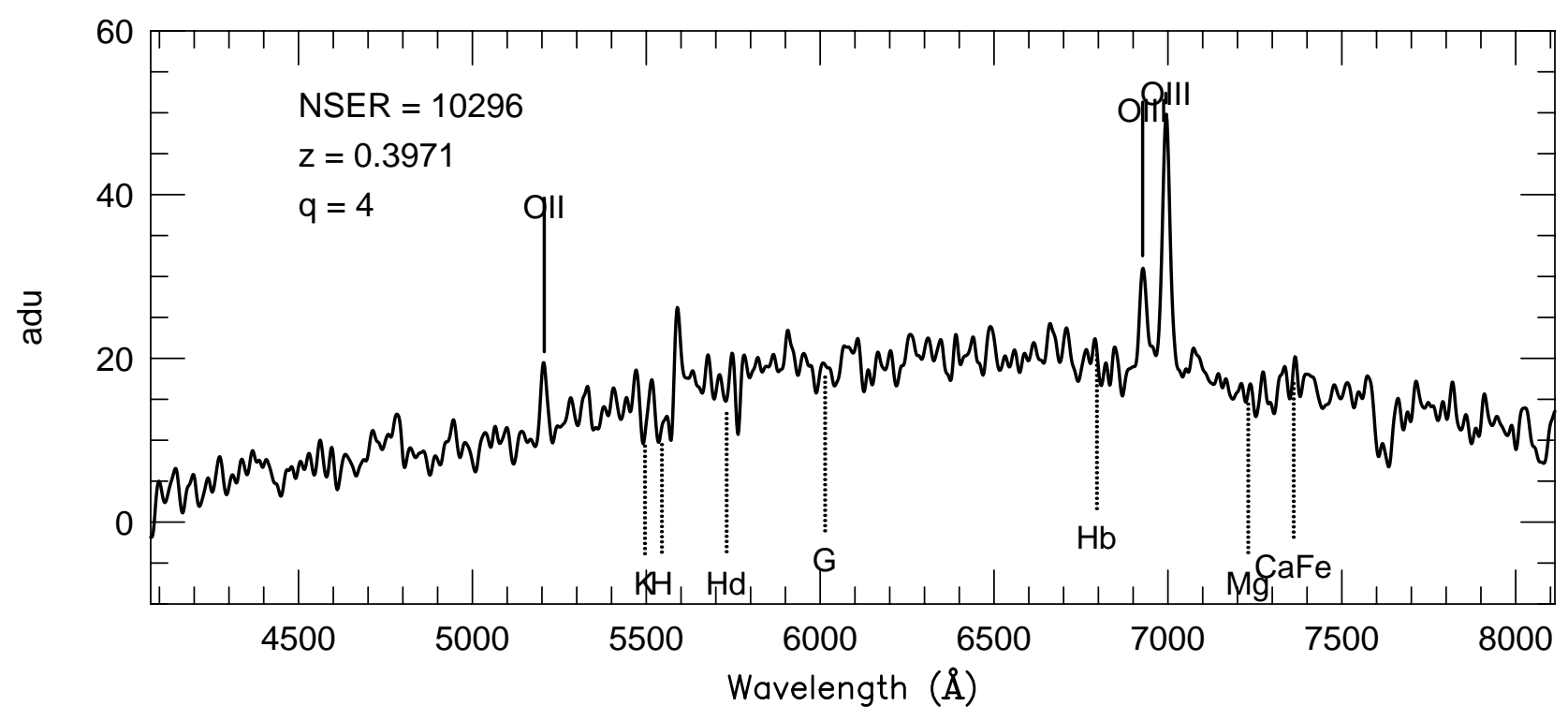




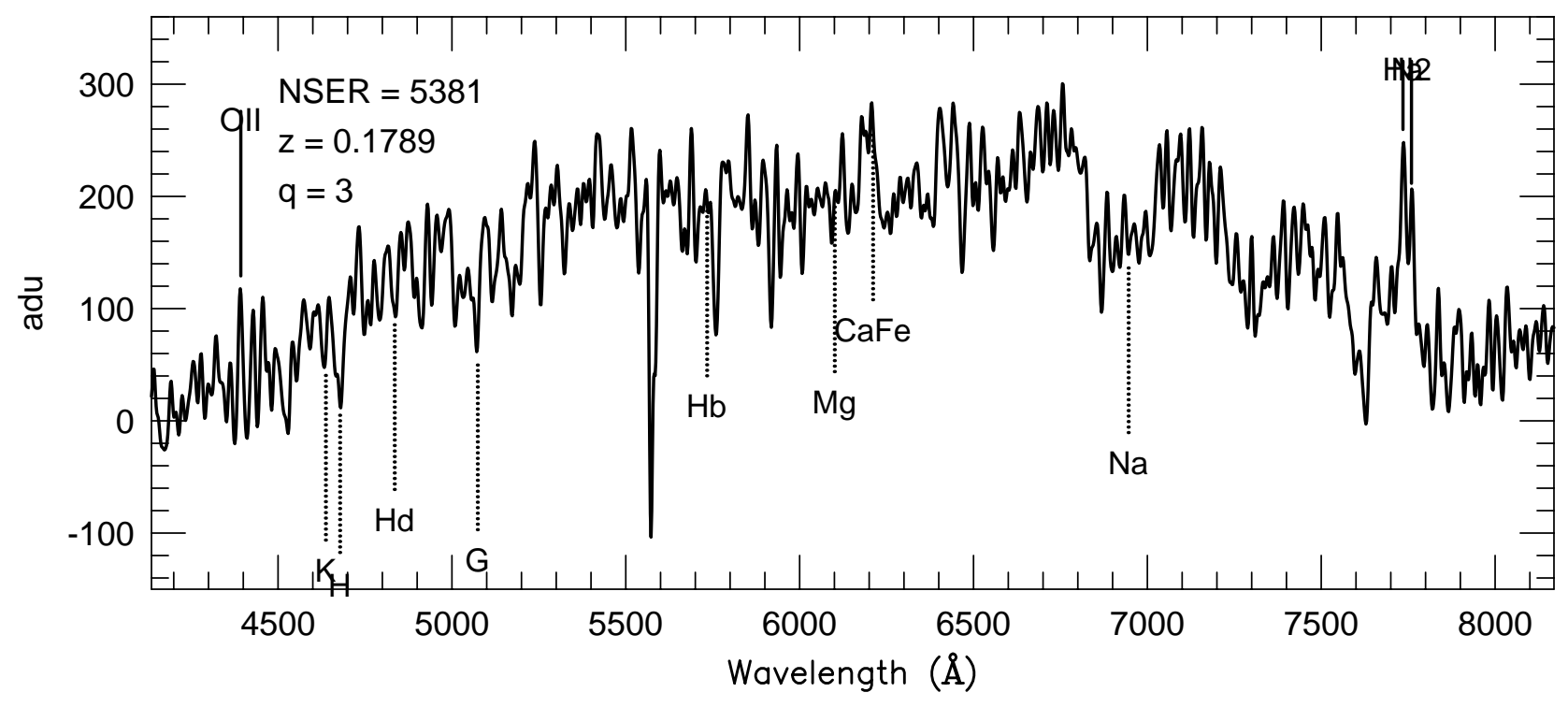




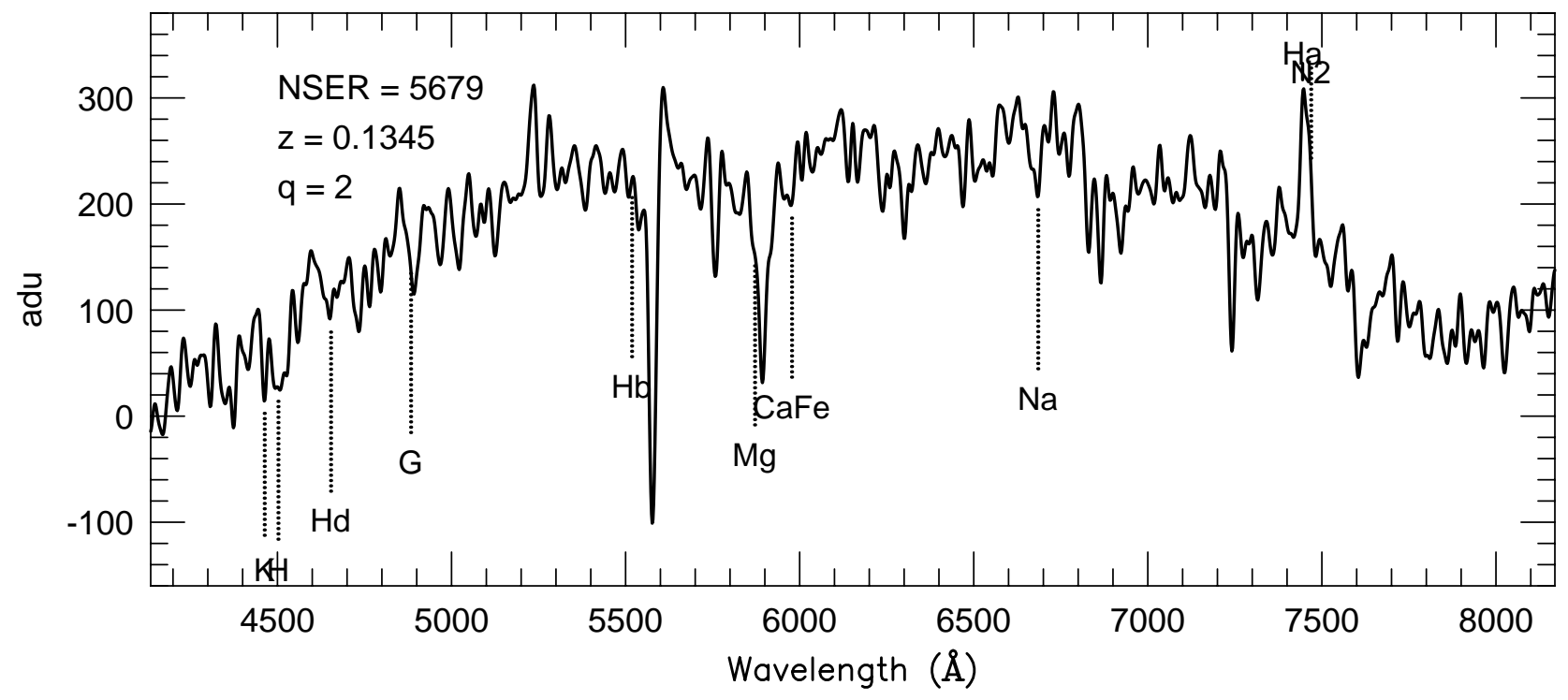




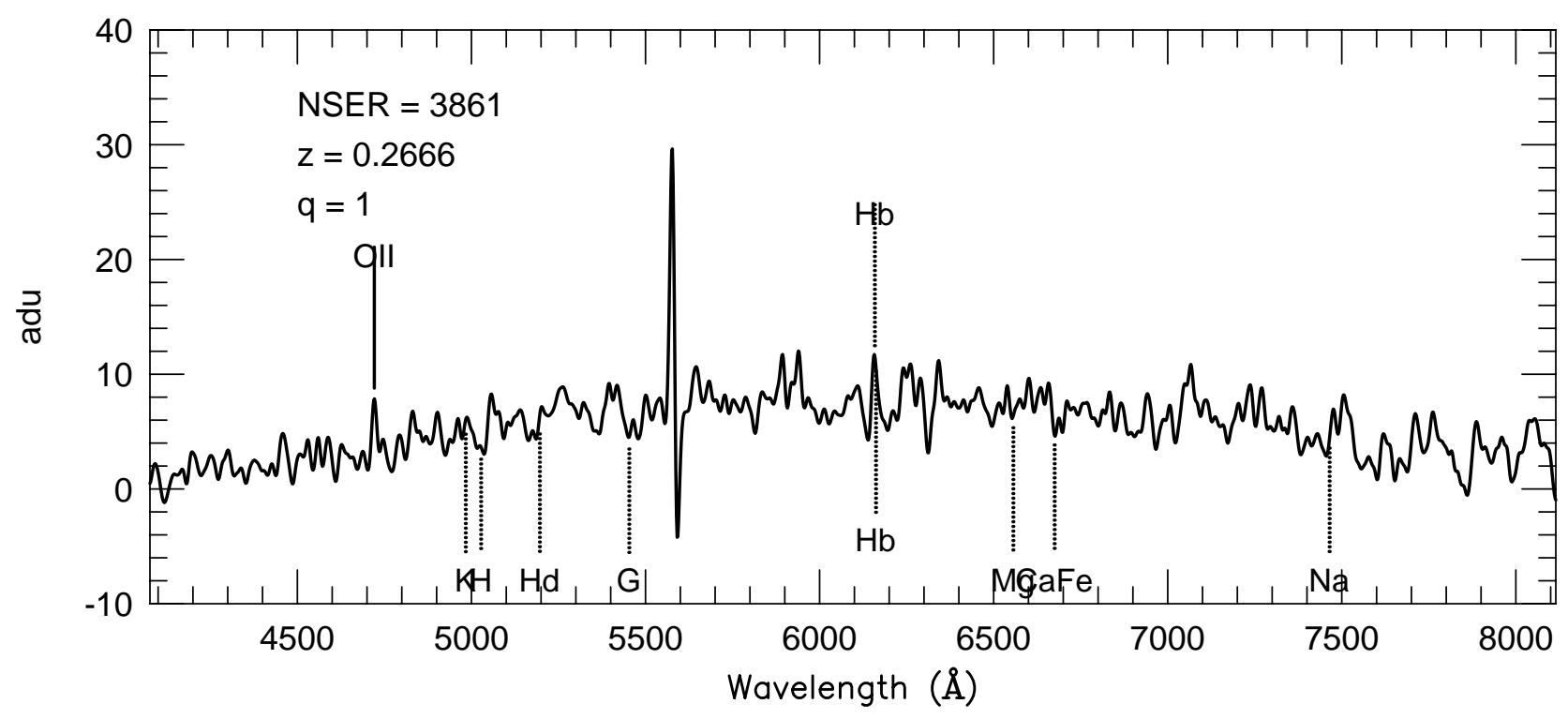




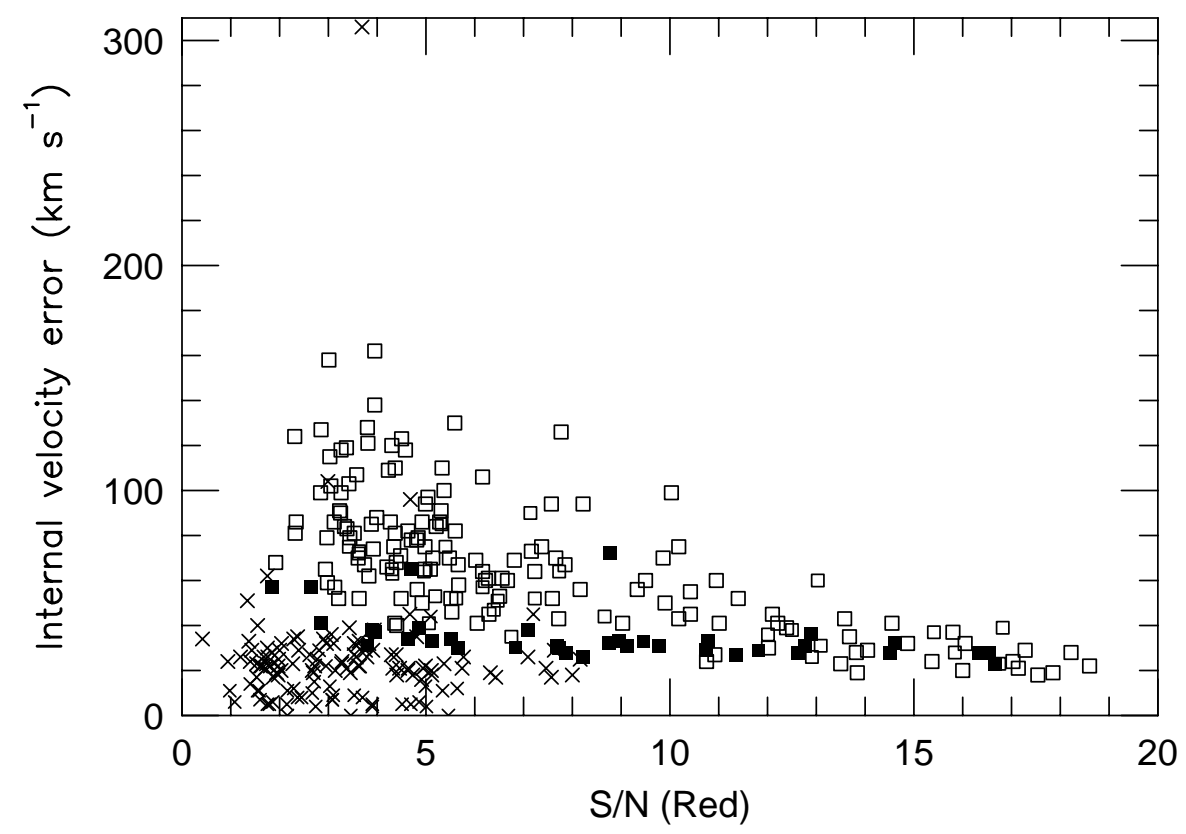




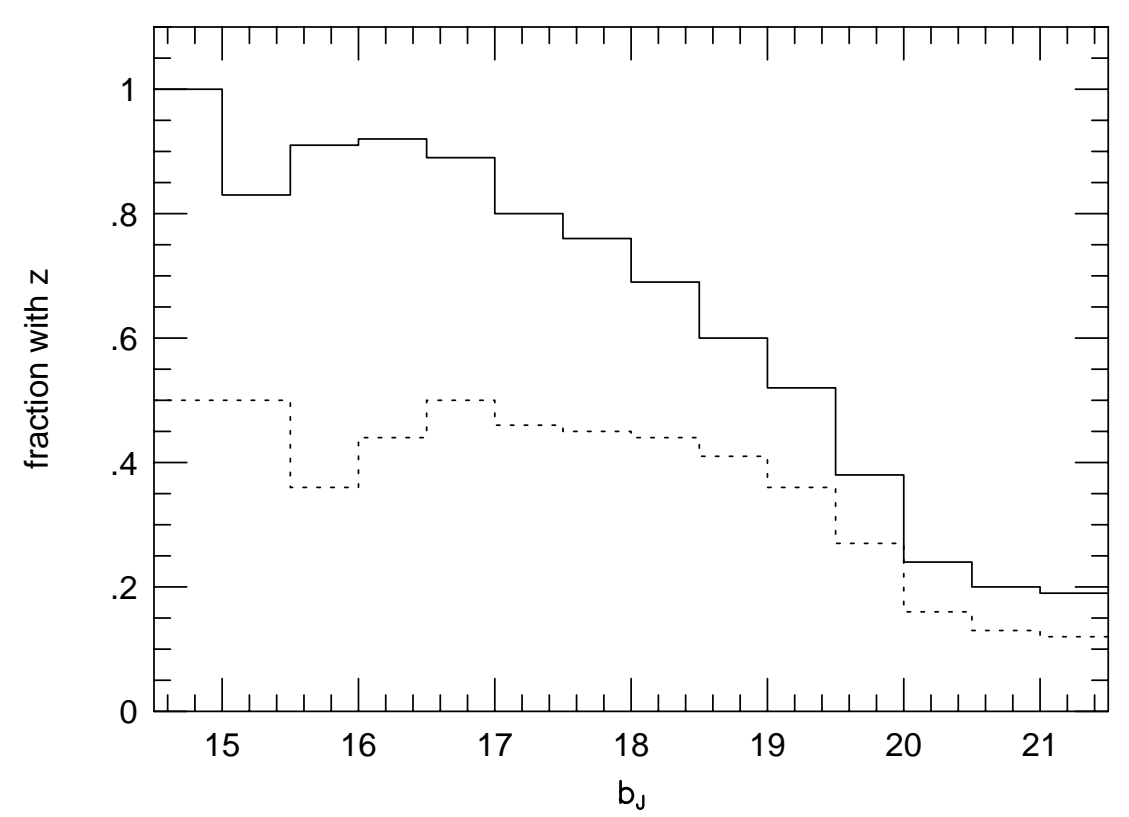




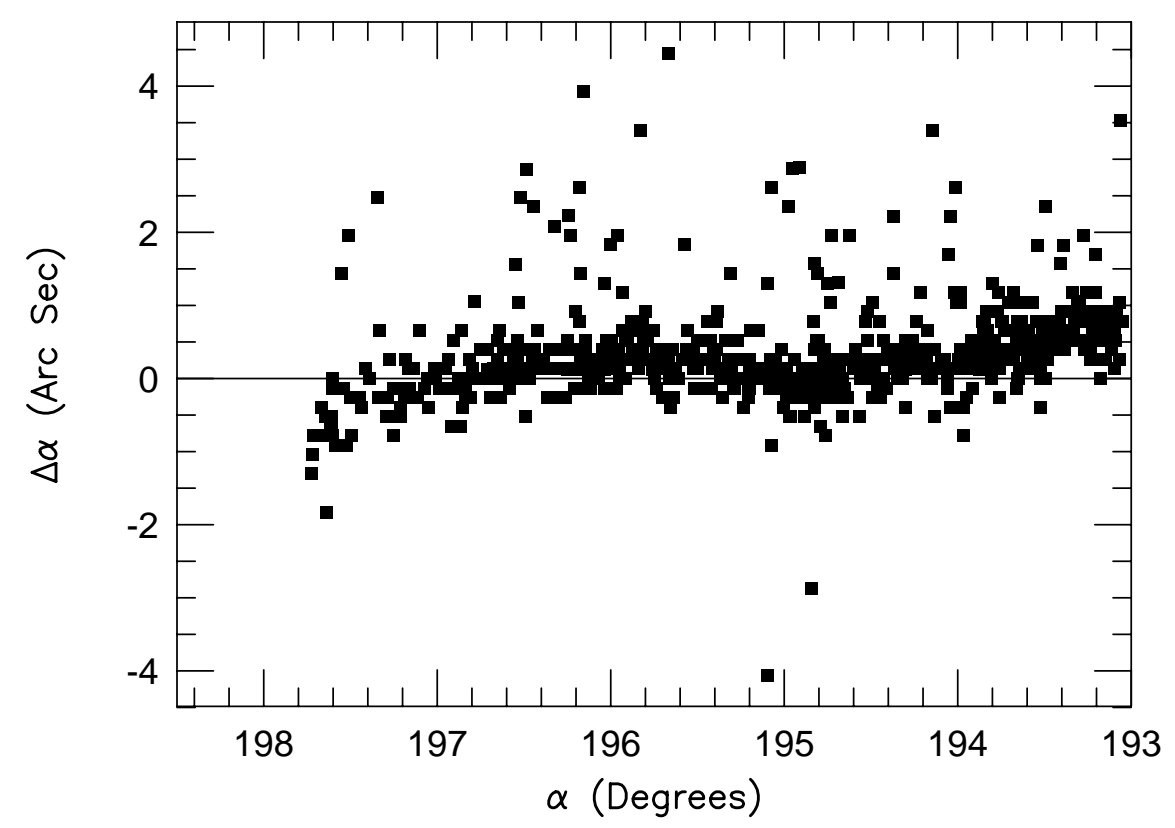




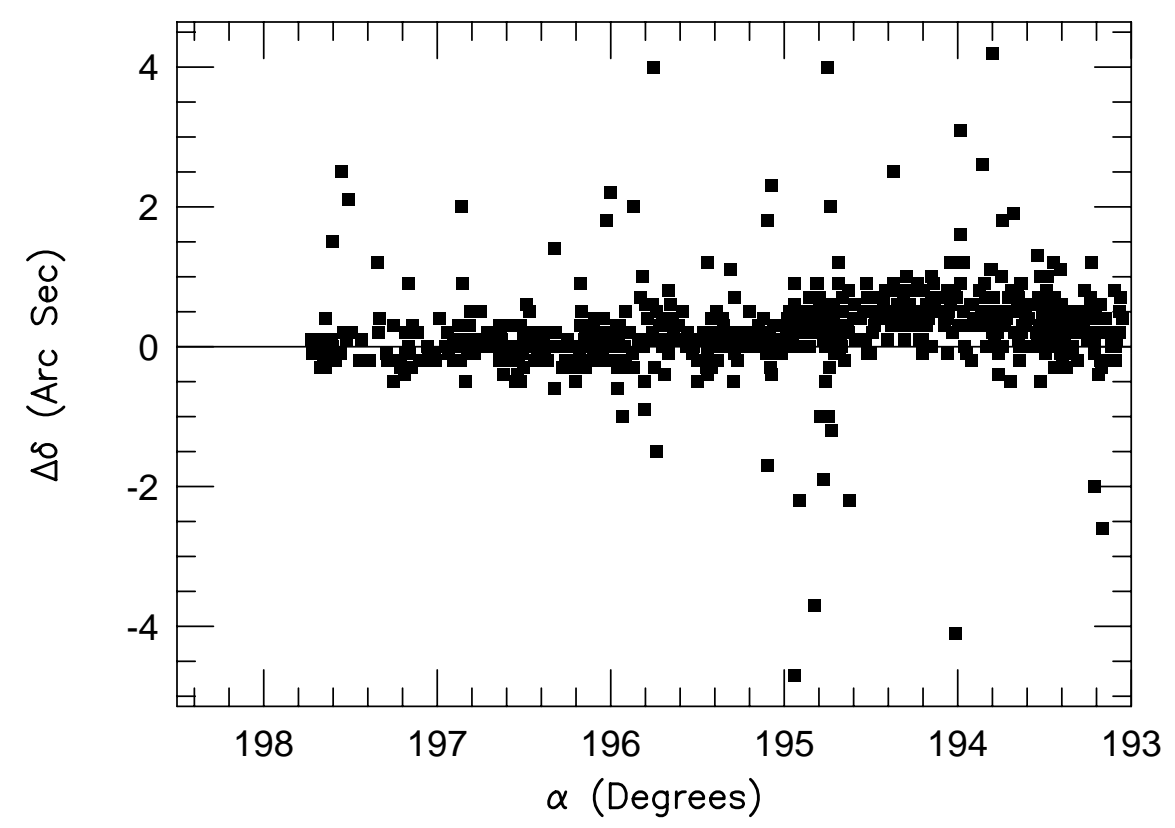




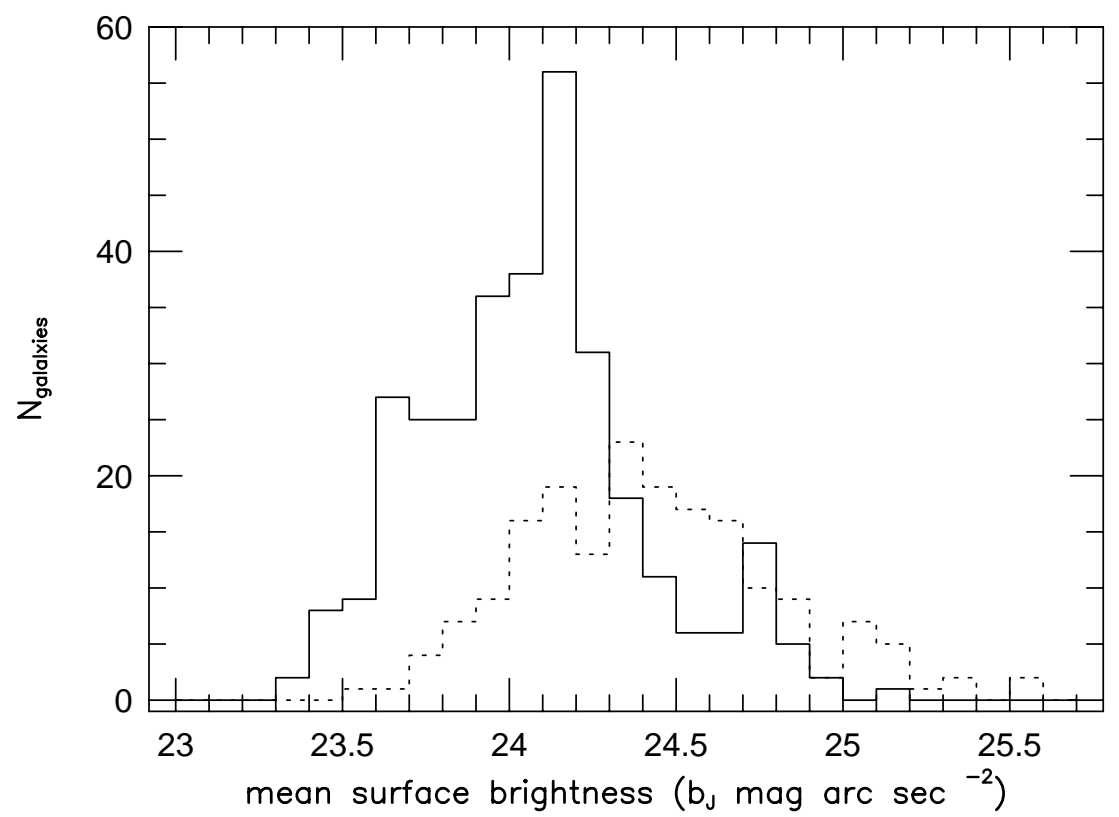




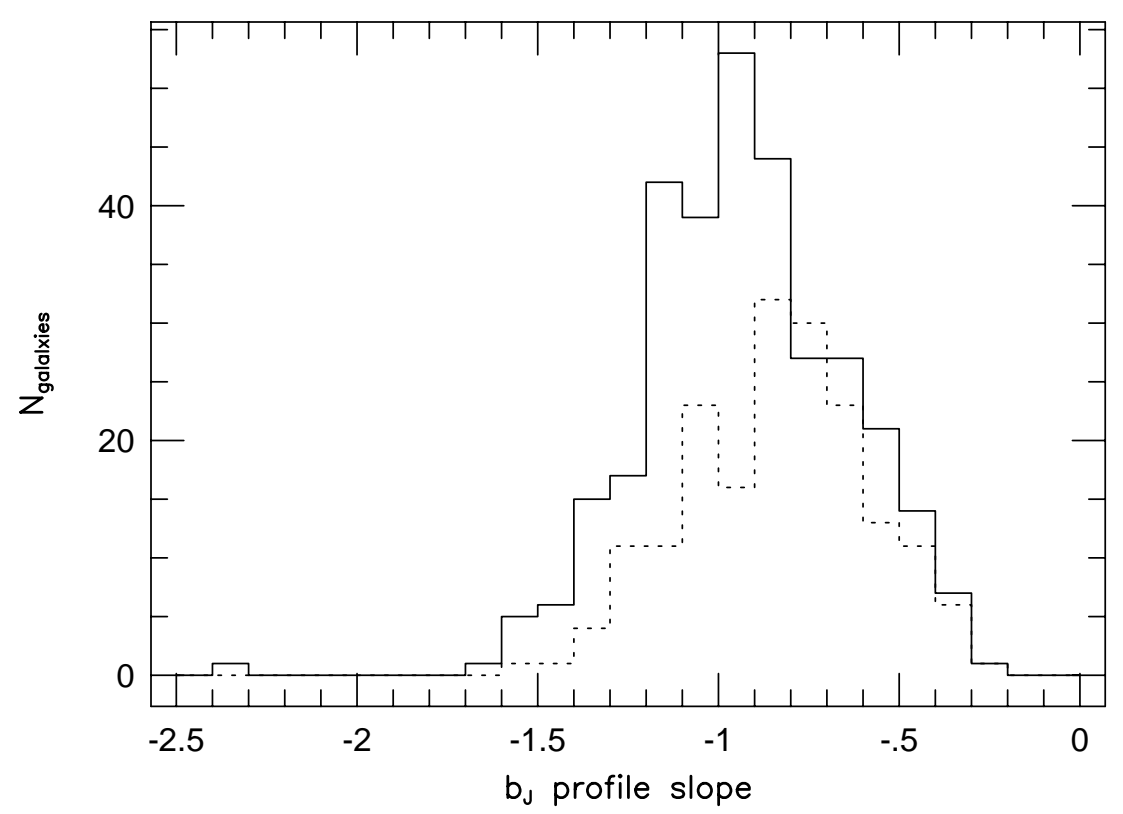




\title{
A Medium-Deep Survey of a Mini-Slice at the North Galactic Pole. II. The Data'
}

\author{
C.N.A. Willmer ${ }^{2,3,4}$ \\ CNRS, Institut d'Astrophysique de Paris, 98 bis, Boulevard Arago, F-75014, Paris, France. \\ David C. Koo ${ }^{2}$ and Nancy Ellman ${ }^{5}$ \\ UCO/Lick Observatory and Board of Studies in Astronomy and Astrophysics, University \\ of California, Santa Cruz, CA 95064. \\ Michael J. Kurtz \\ Harvard-Smithsonian Center for Astrophysics, 60 Garden St., Cambridge, MA 02138. \\ and \\ Alex S. Szalay ${ }^{2}$ \\ Department of Physics, Johns Hopkins University, Baltimore, MD 21218.
}

Received __; accepted _

\footnotetext{
${ }^{1} \mathrm{UCO} /$ Lick Observatory Bulletin 1326

${ }^{2}$ Visiting Astronomer, Kitt Peak National Observatory. KPNO is operated by AURA, Inc. under contract to the National Science Foundation.

${ }^{3}$ Observatório Nacional, Rua General José Cristino 77, Rio de Janeiro, RJ 20921-030, Brazil.

${ }^{4}$ UCO/ Lick Observatory, University of California, Santa Cruz, CA 95064.

${ }^{5}$ Princeton University Observatory, Peyton Hall, Princeton NJ 08544.
} 


\begin{abstract}
We report 328 redshifts, $b_{J}$ magnitudes and $b_{J}-r_{F}$ colors of galaxies measured in a redshift survey of a $4^{\circ} \times 0.67^{\circ}$ slice close to the north Galactic pole. The faintest galaxies in this survey have a magnitude of $b_{J} \sim 20.5$. The redshifts present external errors of the order of $70 \mathrm{kms}^{-1}$, and we estimate that the mean photometry errors are $\sim 0.2$ for magnitudes and $\sim 0.3$ for colors. The redshift completeness level of the sample is of the order of $\sim 35 \%$ at $\mathrm{b}_{J}$ $=20$, and part of this rather low completeness is the result of the combination of limitations imposed by the multifiber system with the clustering of galaxies, and an insufficient number of configurations. At the nominal magnitude limit of the survey, we were able to measure redshifts for $\sim 70 \%$ of the galaxies we observed. From the correlation between observed properties of the galaxies in this sample, we demonstrate that the mean surface brightness is a major limiting factor in our ability to measure redshifts of faint objects.
\end{abstract}

Subject headings: cosmology: observations - galaxies: distances and redshifts galaxies: photometry 


\section{Introduction}

The detection by Broadhurst et al. (1990) of an apparent repetition pattern in the redshift distribution of galaxies toward both galactic poles suggested that structures similar to the walls of galaxies detected by the fully sampled wide-angle surveys of bright galaxies (e.g., da Costa et al. 1988; Geller \& Huchra 1989) were common occurrences. Further evidence supporting this interpretation was presented by Koo et al. (1993), who used several pencil-beam surveys separated by a few degrees close to the direction of the galactic poles demonstrating that the original peaks could not be due to rich groups or clusters of galaxies, while in other directions of the sky similar patterns were also detected. Although the sparsely-sampled pencil beam surveys are an efficient means of probing the galaxy distribution on very large scales, in order to have a better characterization of the properties of these structures, which have a rather low projected surface density, a higher sampling rate than used in the pencil-beam surveys is necessary, as only a few galaxies per wall would be detected by the original probes. In a previous paper (Willmer et al. 1994, hereafter paper I) we presented the results of a redshift survey for a $4^{\circ} \times 0.67^{\circ}$ mini-slice which was designed to characterize the properties of the four or five nearest peaks detected by Broadhurst et al. (1990) in the direction of the north Galactic pole. This work took advantage of the availability in spring 1992 of the HYDRA multi-fiber spectrograph (Barden et al. 1992) which would allow gathering up to 97 spectra simultaneously, thus allowing a denser sampling rate compared to that previously available. The opening angle of this survey was designed to cover a projected distance of $\sim 20 \mathrm{~h}^{-1} \mathrm{Mpc}$ at $\mathrm{z} \sim 0.1(h$ $=\mathrm{H}_{0} / 100 \mathrm{kms}^{-1} \mathrm{Mpc}$ and $\mathrm{q}_{0}=0.5$ assumed throughout), which is the smallest possible projected distance that allows a proper characterization of a structure as a wall of galaxies according to the Monte Carlo simulations carried out by de Lapparent, Geller \& Huchra (1991) and Ramella, Geller \& Huchra (1992). Both works used the Great Wall as a typical representative of walls of galaxies. 
In this paper we report redshifts and magnitudes of galaxies that were observed in this survey, as well as a discussion of the possible selection effects that are present in this sample. The acquisition of the catalog of galaxies and its photometric calibration is described in section 2 , followed by the description of the spectroscopic data in section 3 . The catalog and some of its properties are presented in section 4. Some concluding remarks follow in section 5 .

\section{Astrometry and Photometry}

\subsection{Detection of Objects}

As described in paper I, the sample used in this survey was derived from scans of two recent epoch $(1989,1990)$ Palomar Schmidt plates, SF02410 (IIIaF), and SJ03220 (IIIaJ). These plates had been obtained for the POSS II (Reid et al. 1991), but because of image elongation, they were eventually replaced by higher quality material.

The KPNO PDS microdensitometer was used in density mode to digitize the F plate, using pixels of $10 \mu \mathrm{m}$ sampled at $10 \mu \mathrm{m}$ spacing, each pixel corresponding to $\sim 0.6^{\prime \prime}$ on the sky. An area of about $4^{\circ} \times 1^{\circ}$ centered at $\delta=29.5^{\circ}$ (Epoch 1950) was covered by thirty-five scans of $2000 \times 2000$ pixels, with 200 pixels overlapping both in $\mathrm{X}$ and $\mathrm{Y}$ in order to ensure a uniform magnitude scale and to preserve a global coordinate system. The densities were converted into intensities using the sensitometry spots and the log (exposure) values given by Reid et al. (1991). The fit and transformation were made using the DTOI package of IRAF?. The detection and classification of images was carried out using FOCAS (Jarvis \& Tyson 1981; Valdes 1982). Objects were defined as groups of more than 50 connected pixels

\footnotetext{
${ }^{6} \mathrm{IRAF}$ is distributed by National Optical Astronomy Observatories. NOAO is operated by AURA Inc. under contract to the National Science Foundation
} 
$\left(\sim 23 \operatorname{arc~} \sec ^{2}\right)$ at a threshold of $3 \sigma$ above the sky. From the catalog of objects generated by FOCAS we selected the following parameters for each object: the intensity-weighted $\mathrm{X}$ and Y positions; the isophotal magnitude; the object area; the second inverse moment of the light distribution (Kron 1980); and the automatic classification made by the FOCAS algorithm (stars, galaxies, diffuse, long or noise) which takes into account the shape of the stellar point spread function. The latter was calculated using unsaturated stellar objects detected in each scan.

The brightest stars of the Hubble Space Telescope Guide Star Catalog (Lasker et al. 1990, hereafter referred to as GSC) were identified visually and used to define an initial astrometric solution. Subsequently, all detected objects contained in the GSC were identified and used to obtain the final solution, which was therefore in the GSC reference frame. The catalog derived from FOCAS, which was used for the first spectroscopic run, had equatorial positions derived from this solution.

A new astrometric solution was carried out to verify whether imprecision or systematic effects in the astrometry could have been a possible cause of the rather low success rate in the spectroscopy during the 1992 run (see section 4 below). The first step in this new solution involved defining a new primary astrometric reference frame, which was done by measuring the positions of a total of 67 stars from the ACRS catalog (Corbin \& Urban 1988), which has positions better than 0.1", on the Lick astrograph plate Ay-07697 (epoch 1974) using the Lick Observatory Automatic Measuring Engine (AME, Klemola, Jones \& Hanson 1987). The second step was the redetermination of the right ascension and declination on the astrographic plate of GSC stars that defined the secondary frame of reference. The solution for the secondary reference frame used 442 stars and has rms residuals of the order of $0.3^{\prime \prime}$. A comparison between our measurements and those in the GSC is shown in Figure 1, where a systematic difference of up to $1^{\prime \prime}$ is easily seen, and the 
mean residuals between coordinates are shown in Table 1. The "corrected" coordinates of GSC stars were then used to convert the scan X and Y coordinates of FOCAS objects (referred to the origin of the first scan) into equatorial coordinates. The rms residuals for this transformation are of the order of $0.5^{\prime \prime}$, and the total number of objects above detection limits in the PDS-derived catalog is 13886.

Both plates were scanned by the Automatic Plate Measuring (APM) system (Kibblewhite et al. 1984) at the Institute of Astronomy, University of Cambridge. The APM is a high-speed microdensitometer that uses a scanning laser to measure the plate transmission, and allows either detecting and classifying objects in real-time when in catalog mode, or obtaining a digitized image when in raster mode. The APM scans were carried out by M. Irwin and N. Ellman in catalog mode, using a pixel size of $8 \mu \mathrm{m}\left(0.54^{\prime \prime}\right.$ on the sky) and followed the standard procedure of detection of objects. As described by Maddox et al. (1990) and Infante \& Pritchet (1992), the data acquisition with the APM is made in two steps. First, the plates are scanned to determine the local sky background in each region of the plate, which is done by dividing the plate into a grid of $64 \times 64$ pixels; for each square of this grid, the sky background is determined by fitting the histogram of pixel values and determining the value of the mode. From the ensemble of these squares the sky background at each pixel position is estimated by means of a bilinear interpolation (Maddox et al. 1990).

In the second pass objects are identified as groups of more than 12 connected pixels ( $3.2 \operatorname{arc} \sec ^{2}$ ) with densities higher than $2 \sigma$ above the sky background. This corresponds to a limiting surface brightness of about $25.5 \mathrm{~b}_{J}$ magnitudes $\operatorname{arc~sec}^{-2}$ and $24.5 \mathrm{r}_{F}$ magnitudes arc $\mathrm{sec}^{-2}$ (Ellman 1994). The limiting magnitude of these plates were estimated by Ellman to be $b_{J} \sim 23.5$ and $\mathrm{r}_{F} \sim 22.2$. A series of parameters are calculated for each object: the density-weighted $\mathrm{X}$ and $\mathrm{Y}$ centroid positions; the integrated isophotal density, which 
corresponds to the APM "magnitude"; the intensity-weighted second order moments ( $\mathrm{S}_{x x}$,

$\left.\mathrm{S}_{x y}, \mathrm{~S}_{y y}\right)$ from which the size, shape and position angle of objects may be derived; the peak density; and the number of pixels above eight preset density threshold levels. The equatorial coordinates are obtained from the positions of several PPM stars (Röser \& Bastian 1991) and the final positions are usually accurate to about $0.5^{\prime \prime}$ (Maddox et al. 1990). These parameters are combined into a series of classifiers which allow an objective separation between stars, galaxies, merged images, and noise. The final catalog is then output with equatorial coordinates, classifications, and the set of parameters described above.

\subsection{Combination of Catalogs}

In this work we used APM data in a region slightly larger than our survey, covering the whole R.A. range of the plates in the declination range $29^{\circ} \leq \delta \leq 30^{\circ}$ (Epoch 1950.0). The total number of detections in each sub-catalog is 36619 for the F plate and 36232 in J. Combining these catalogs it became possible to estimate the astrometric uncertainties, eliminate spurious detections, and have an independent object classification and an estimate of the color. Although small systematic differences in equatorial coordinates dependent on the position on the plate were detected, these are small and are always less than $0.3^{\prime \prime}$ (Ellman 1994), mostly in right ascension. This variation was taken into account through the use of a linear fit when the combined catalog was made.

The master catalog used in this survey was defined from the J plate scan, and although this choice was arbitrary, the smaller number of objects down to the detection limit suggested that by using it, the number of objects without colors might be minimized. Besides the cut in declination, a further cut in apparent magnitude, corresponding to approximately $\mathrm{b}_{J}=21.5$ was applied. This was faint enough that no objects would be lost when defining the redshift survey sample, yet bright enough that most of the objects were 
real and would minimize the number of multiple matches for a same object. No cut in magnitude was applied to the $\mathrm{F}$ plate catalog in order to maximize the number of objects with colors. A match was accepted whenever the separation (after correcting for the linear term described above) between objects in both catalogs was less than 1.25". This small separation ensured that most objects would have only one match. Further searches were made for non-matching objects at separations of $2^{\prime \prime}, 3^{\prime \prime}, 4^{\prime \prime}$ and $5^{\prime \prime}$, in order to include objects that are very bright or that could be components of images classified as merged in one of the APM catalogs. The combined catalog contains a total of over 13000 objects, of which about $10 \%$ were classified in either or both of the APM catalogs as merged.

This combined catalog was then correlated with that derived from the PDS scans. There were three motivations for this: (1) it would allow an independent verification of the astrometry; (2) it would allow comparing independent classifications of objects, and (3) it enabled us to separate merged images. One of the features of FOCAS is that it allows splitting merged images by making cuts at higher isophotal levels, which improves the coordinates of objects at the possible expense of magnitude accuracy. This of course depends on the relative apparent magnitudes of the objects making up the merger. The total of matching objects is 7710 , and the comparison of coordinates resulted in mean differences of the order of $0.3^{\prime \prime}$ (see Table 1). Of the 1162 objects classified as merged in the APM J catalog, 604 could be split through this procedure. The rather small number of matches is the result of a combination between the larger angular coverage of the APM scans $\left(5^{\circ}\right)$ relative to the PDS scans, added to the fact that no magnitude cut was applied to the PDS catalog, which reaches deeper than the adopted cut in $\mathrm{b}_{J}$.

The final catalog of objects after matching with the PDS scans contains 13524 objects. The object class in this catalog combines the classification from each of the scans and attributes a score that ranges from high probability of being a star or galaxy, when the 
object presented the same classification in all catalogs, to uncertain. This classification also took into account cases in which an object was not contained in one of the scans of the F plate. All objects presenting either conflicting classifications or that were classified as merged and brighter than $\mathrm{b}_{J}=20.5$, were inspected visually and classified using the images of PDS scans.

Further checks on the astrometry of this combined catalog were carried, and are also presented in Table 1. We have compared the APM coordinates with two unpublished catalogs of the SA 57 region, the first coming from Kron (1980) which contains the photometric corrections of Koo (1986), that we will refer to hereafter as the KK catalog, and that of S. Majewski, which, although derived from KK, has a greater astrometric precision. From the comparison with the catalogs in SA 57 we can see that our coordinates have an accuracy of about $0.4^{\prime \prime}$. The differences we find with the KK catalog have a systematic component, while the comparison with Majewski's catalog shows only random errors. In addition to these catalogs, which contain mainly faint objects, we compared our positions with two surveys of bright galaxies, that of the drift scan of Kent, Ramella \& Nonino (1993, hereafter KRN), and the catalog of van Haarlem et al. (1993), which was also derived from APM photometry. The comparison with the KRN catalog showed 96 galaxies in common. By considering the values quoted by KRN for their positional uncertainty (0.5 " in R.A., 0.9 " in decl.), our errors would be of the order of 0.41 " in R.A. and 0.87 " in decl. The latter is much larger than what is inferred from the comparison with the catalogs of fainter galaxies. If we assume that the APM coordinates are good to about $0.4^{\prime \prime}$, this result implies that the uncertainty in declination in KRN could be underestimated. However, another possibility is that our measurement of the centroids of bright galaxies could be affected by effects such as saturation. This interpretation would also explain the rather large dispersion in our comparison with the van Haarlem et al. (1993) sample, although, in this case, we should stress that the number of objects in common is very small (19 galaxies). 


\subsection{Photometry}

Although we had originally intended to calibrate the plates using CCD photometry, which was obtained with the Mount Hamilton $1.0 \mathrm{~m}$ Nickel Telescope, because of poor weather during our photometry runs, only a very small number of calibrating objects were measured, and, even for those, the quality of the data would not allow an adequate calibration. Therefore, we have to rely on photographic data, and in this calibration we have followed the procedure described by Ellman (1994).

As the catalogs produced both by APM and FOCAS contain isophotal magnitudes, in order to obtain physically meaningful colors, it is necessary to measure "total" magnitudes for galaxies, or to use the same metric aperture in all bands (e.g., Bershady et al. 1994). A previous attempt to estimate total magnitudes for APM-generated catalogs was carried out by Infante \& Pritchet (1992), who used the size information provided by APM to divide galaxies into different surface-brightness classes and then used this information to correct to total magnitudes. However, as discussed by Ellman (1994), this procedure could introduce discontinuities for objects close to the surface brightness class limits. Therefore, in her thesis, Ellman used a continuous correction that depended on the size of an object relative to the size of a star with the same magnitude. This correction was determined by fitting the stellar locus of the size-magnitude diagram for each color with a low-order polynomial, and then using this correction when solving the photometric transformation.

To calculate the photometric transformation, we used the catalog of KK, which contains total magnitudes measured in several bands. Although this catalog is derived from photographic photometry, by using it one can somewhat offset the lower precision of the photographic photometry against the larger color corrections that would be necessary if CCD photometry were used. In the calibration we used only objects classified as galaxies in both APM catalogs and KK and that matched within $2^{\prime \prime}$ of the KK objects after the 
correction for small systematic offsets, calculated using the catalog of Majewski. The total number of galaxies used in the transformation was 115. These transformations were solved using the IRAF PHOTCAL task:

$$
\begin{aligned}
& b_{a p m}=b_{1}+b_{2} \mathrm{~b}_{J}+b_{3}\left(\mathrm{~b}_{J}-\mathrm{r}_{F}\right)+b_{4} S c_{J} \\
& r_{a p m}=r_{1}+r_{2} \mathrm{r}_{F}+r_{3}\left(\mathrm{~b}_{J}-\mathrm{r}_{F}\right)+r_{4} S c_{F}
\end{aligned}
$$

where we have followed Ellman (1994) in considering a size correction in the transformation $\left(\mathrm{Sc}_{J}\right.$ and $\mathrm{Sc}_{F}$ respectively, where $\left.\mathrm{Sc}=\mathrm{S}_{x x}+\mathrm{S}_{y y}\right)$. As shown by Ellman (1994), the color term in the $b_{a p m}$ transformation (eq. 1) is negligible, and this transformation was solved setting $b_{3}=0$. The rms dispersion is $\sim 0.12$ mag. By comparing measurements of objects classified as galaxies in the KK sample, but not necessarily in the APM catalogs, we find a mean difference of

$$
\begin{gathered}
\mathrm{b}_{J}-b_{K K}=0.002 \pm 0.177 \\
\mathrm{r}_{F}-r_{K K}=-0.006 \pm 0.168 \\
\left(\mathrm{~b}_{J}-\mathrm{r}_{F}\right)-\left(b_{K K}-r_{K K}\right)=0.009 \pm 0.149
\end{gathered}
$$

for 214 objects matching within $2^{\prime \prime}$. A comparison between the photometry in both catalogs is shown in the panels of Figure 2. Figure 2(a) shows the difference $b_{K K}-\mathrm{b}_{J}$ versus $b_{K K}$. For magnitudes fainter than $\mathrm{b}_{J}=19.0$ the measurements are quite similar, but at brighter levels, saturation causes our magnitudes to be fainter than those in the KK catalog. Figure 2(b) shows the behavior of color as a function of magnitude, and we can see that there is a tendency of brighter objects to be redder in our sample, the expected behavior in the case of our measurements being primarily affected by saturation in the J plate.

In order to have an independent check on the quality of our photometry, in particular to search for the presence of systematic effects dependent on position, we have compared 
our $\mathrm{r}_{F}$ photometry measurements with those of KRN. The following transformation (e.g., Ellman 1994) was calculated:

$$
r_{K R N}=r_{1}+r_{2} \mathrm{r}_{F}+r_{3}\left(\mathrm{~b}_{J}-\mathrm{r}_{F}\right)+r_{4} \theta
$$

where $\theta$ is the distance from the plate center in arc minutes. We find that the dependence on distance from plate center is very small $(0.004 \pm 0.004 \mathrm{mag})$, a result which is of the same order as found by Ellman (1994), who used independent CCD photometry in the B and $\mathrm{V}$ bands. There is no significant improvement in the fit by considering the color term. The final comparison kept both terms constant and equal to zero, giving an rms of 0.20 mag, with a slight slope $\left(r_{2}=1.14\right)$.

From these comparisons, we estimate that the photometry errors are of the order of 0.2 mag, which added in quadrature imply that the errors of the color should be of the order of $0.3 \mathrm{mag}$.

The final procedure that was carried out was to inspect the APM areal profiles in order to estimate the number of saturated pixels for each object. As shown by Ellman (1994), it is possible to estimate where the emulsion becomes non-linear by inspecting a diagram of peak intensity versus magnitude. By using the same values as Ellman (1994), we find that the total number of galaxies that have pixels above emulsion non-linearity in our catalog is 292. In Figure 3 we show the distribution of the number of galaxies in bins of $0.2 \mathrm{mag}$; Figures 3(a) and 3(b) show the logarithm of total count of galaxies represented as solid lines, while the dotted lines represent galaxies presenting saturated pixels in the J and $\mathrm{F}$ plates respectively. For the blue plate, the number of galaxies presenting saturation is $\sim 80 \%$ at $\mathrm{b}_{J}=18.0$, while at $\mathrm{b}_{J}=19.5$ there are very few galaxies with pixels above non-linearity, and for most of these, only 1 or 2 pixels are saturated, so it is likely that the magnitudes are not very far off. For the $\mathrm{F}$ plate the corresponding limits are 16.5 for $80 \%$ of galaxies with unreliable magnitudes, while at 18.0 there are no more galaxies with 
saturated pixels. We should note that the worst cases for the F plate are galaxies presenting $4 \%$ non-linear pixels.

\section{Spectroscopy}

The spectroscopic observations were made with the KPNO Mayall $4.0 \mathrm{~m}$ telescope during two runs in 1992 February 27-29 and 1993 February 15-17. Because of bad weather on both runs, the total observing time was equivalent to only 2 useful nights. The objects were observed using the HYDRA multi-fiber positioner (Barden et al. 1992) and a bench-mounted spectrograph, with the KPNO T2KB chip. This setup makes it possible to obtain spectra of up to 97 objects simultaneously. We used the red fiber cable, and the wavelength ranged from 4100 to $8100 \AA$ with a dispersion of $2 \AA$ per pixel and resolution of about $8 \AA$ FWHM. Typically $\sim 75$ fibers were assigned to objects, while about 15 were assigned to "empty" sky positions. The sky positions were selected by dividing the survey region into a grid of $20^{\prime \prime}$ squares and then counting the number of objects within these squares using the full APM catalogs (i.e., both catalogs without matching and without any magnitude or object class cut). In this way, we optimized the chance of not having the sky fibers contaminated by faint background objects, as well as allowing a large choice of possible sky positions; for a given field, there were typically about twice as many sky positions as there were objects. The fiber assignment was made though a code written by P. Massey which optimizes the number of fibers assigned to objects taking into account the number of required sky positions, the number of "field orientation probes" (that allow verifying the astrometry and orientation of the field, but are also useful to check the guiding or presence of clouds), and an optional weighting scheme, which is based on the rank of the object in a catalog. For our runs we used the "strong" weighting scheme on a magnitude-ordered catalog. During the second run, we could improve the fiber assignment through the use 
of an optional feature that allowed the allocation of fibers visually. Integration times were usually $6000 \mathrm{~s}$ divided into three exposures, in order to allow an efficient removal of cosmic-ray events. Comparison exposures were taken before and after each night to provide the wavelength calibration and to track shifts in the positions of comparison lines. During both runs we observed spectrophotometric and radial velocity standard stars as well as a few bright galaxies, which could serve as templates for the redshift determination, and to determine the radial velocity uncertainties and zero points. Data were reduced in the IRAF environment following standard procedures (e.g., Massey 1992) with removal of bias and dark current prior to the combination of images and extraction of spectra. It was also found that removing scattered light improved the final results, so that prior to the extraction of spectra, this correction was done. In the IRAF tasks dealing with multi-fiber data, flat-fielding is usually carried out during extraction of the spectra. The spectra were extracted using the DOHYDRA task written by F. Valdes. The wavelength solution is also determined within this task, and typically about $35 \mathrm{He}-\mathrm{Ne}-\mathrm{Ar}$ lines were used in the final transformations with an rms residual usually better than $0.10 \AA$. The last step in this reduction is the sky subtraction which used an average sky spectrum obtained combining the sky exposures of a given setup. Redshifts were measured using the RVSAO package (Kurtz et al. 1992) which measures redshifts using cross-correlation and emission-line analyses. For the cross-correlation analysis, a series of templates was used, which included the standard CfA templates distributed with RVSAO; composite spectra of stars and galaxies measured for the Southern Sky Redshift Survey (da Costa et al. 1989); high signal-to-noise ratio spectra of M31 (kindly provided by C. Bellanger and V. de Lapparent) and NGC 7507; spectra of radial velocity standard stars observed with HYDRA; and finally, composite spectra of standard stars of Jacoby, Hunter, \& Christian (1984) combined into different luminosity and spectral classes. This assortment of templates permits optimizing the cross-correlation peak. For all spectra we obtained, the final redshifts produced by 
RVSAO were only accepted (or rejected) after visual inspection. The internal errors for the redshift are estimated from the ratio between the cross-correlation peak relative to the average noise peaks, or from the dispersion between the radial velocities of each emission line. When both are present, these errors are combined (e.g., Tonry \& Davis 1979). We should stress that these errors are certainly underestimated so that external estimates of the uncertainties are required.

In the panels of Figure 4, we show some spectra of objects for which we could measure redshifts. The first five, Figures 4(a) through 4(e), are typical representatives of objects for which we could measure cross-correlation velocities, and the spectra are ordered following decreasing redshift quality (see Section 4.1 below). Figures 4(f) through 4(j) show the analogous case for objects presenting emission lines. None of these spectra were corrected for the instrumental response. We show for each object its identification, redshift, and redshift quality from Table 3 and, in the case of Figure 4(e), from Table 5. We also show the expected positions of some prominent absorption lines, which are used in the visual confirmation of the redshift, as well as emission lines that were either used for measuring the redshift or that would allow a further confirmation on the correctness of the redshift.

In general, the mininum signal-to-noise ratio $(\mathrm{S} / \mathrm{N})$ for which cross-correlation velocities could be obtained was 3 , the $\mathrm{S} / \mathrm{N}$ being measured in the interval between 6000 and 6200 $\AA$, where the spectra are generally flat and where there is no sky emission. In Fig. 5 we show the a plot of $\mathrm{S} / \mathrm{N}$ versus internal redshift uncertainty, where we discriminate objects that produced both cross-correlation and emission line velocities by using solid squares; objects that produced cross correlation only are shown with open squares, and objects that produced emission lines only are shown with crosses. We can see that emission line velocities could be measured down to very low values of $\mathrm{S} / \mathrm{N}$, while very few objects presented cross-correlation velocities below $\mathrm{S} / \mathrm{N}=3$; above $\mathrm{S} / \mathrm{N}=9$, all objects presented 
a cross-correlation velocity. We can see that the internal errors are in their vast majority less than $100 \mathrm{kms}^{-1}$. In general, the cross-correlation velocities obtained from different templates usually agreed within the estimated internal errors.

To estimate our external uncertainty, we have compared our radial velocities with measurements obtained by Ellman (1994), van Haarlem et al. (1993), ZCAT (Huchra 1993) and the radial velocity standard stars we observed. These measurements are presented in Table 2, and the column descriptions may be found in the table notes. With ZCAT (Huchra 1993), there are 15 galaxies with redshifts in common (not 13 as reported in paper I), with a mean difference of

$$
v_{\text {here }}-v_{Z C A T}=-3.7 \pm 73.5 \mathrm{kms}^{-1}
$$

With the catalog of van Haarlem et al. (1993), there are 9 galaxies for which we have also measured radial velocities; the mean difference we find is

$$
v_{\text {here }}-v_{v H}=-33.3 \pm 66.2 \mathrm{kms}^{-1} \text {. }
$$

With the 5 galaxies in common with Ellman (1994) we find

$$
v_{\text {here }}-v_{\text {Ellman }}=-122.4 \pm 41.1 \mathrm{kms}^{-1} .
$$

A $21 \mathrm{~cm}$ velocity has been obtained for one of our faintest galaxies (M.A.G. Maia, private communication), which gives a difference of $\sim-60 \mathrm{kms}^{-1}$. The radial velocity standard stars were selected from the Astronomical Almanac (Blumberg \& Boksenberg 1995) and we find for 6 measurements

$$
v_{\text {here }}-v_{A A}=-9.4 \pm 13.6 \mathrm{kms}^{-1} \text {. }
$$

For all galaxies and stars, which make up a total of 35 objects, we get

$$
v_{\text {here }}-v_{\text {others }}=-30.2 \pm 69.8 \mathrm{kms}^{-1} \text {. }
$$


This suggests that our external errors are probably less than $100 \mathrm{kms}^{-1}$, although there is a possibility that we might have a zero-point shift of a few tens of $\mathrm{kms}^{-1}$.

\section{Properties of the Redshift Catalog}

\subsection{Object Catalogs}

The total number of objects within the limits of this sample (from $\sim 12^{h} 47^{m}$ to $\sim 13^{h}$ $16^{m}, 29^{\circ} \leq \delta \leq 30^{\circ}$ ) is 13524 , of which 7481 are stars, 4242 are galaxies, 137 are merged images and 1664 are noise. The latter were not removed a priori from the catalog, because in a few cases these corresponded to images of bright objects. For the observations, as well as the analyses, we considered the region in the range $12^{h} 52^{m} \leq \alpha \leq 13^{h} 12^{m}$ and $29^{\circ} 05^{\prime} \leq \delta \leq 29^{\circ} 54^{\prime}$ (epoch 1950.0). The range in declination was chosen because it corresponds to the field size that could be observed with HYDRA, while the R.A. range corresponds to the smallest survey opening angle that could allow determining the presence of wall-like structures at $\mathrm{z} \sim 0.1$; at this distance the opening angle corresponds to $\sim 20$ $h^{-1}$ Mpc. At the nominal magnitude limit of the survey $\left(b_{J}=20\right)$, there are 1013 galaxies, 11 merged images and 3097 stars in the catalog.

The total number of redshifts we measured is 347, 326 of which are of galaxies, 2 of which are quasars and 19 (5\%) of which are stars. During the observations, objects fainter than the nominal limit were also considered as possible targets to place fibers, so that the catalog contains redshifts of 47 galaxies fainter than $\mathrm{b}_{J}=20$. The catalog of observed galaxies is presented in Table 3, and the columns are described at the end of the table. We should note also that for the quasars the redshifts were estimated visually, as we were not successful in fitting these lines with the RVSAO software. The estimate of the mean surface brightness was calculated as $\mathrm{SB}=\mathrm{b}_{J}+2.5 \log _{10}$ (area), the area having been derived 
from the total number of pixels contained by the outermost isophote detected by APM transformed into square arc seconds. This expression is not entirely correct, in the sense that the magnitude we are considering is a total magnitude and not the isophotal magnitude measured within the limiting isophote used by APM. However, we feel this should serve as an adequate estimator of the surface brightness. The calculation of the surface brightness within a circle of $2^{\prime \prime}$ diameter, corresponding to the approximate size of the fibers projected on the sky, while more meaningful, would be affected by large uncertainties, as the catalog output by the APM contains the measurement of the number of pixels above 8 preset density levels. This means that it would be necessary to integrate the fitted image profile in order to estimate the luminosity within $1^{\prime \prime}$ radius. This estimate would also have to take into account the characteristic curve as well as make a correction for saturated pixels.

For completeness, in Table 4 we present a list with radial velocities and magnitudes of objects in our catalog that turned out to be stars. In addition to the 335 objects presented in Tables 3 and 4, we present in Table 5 a list of objects ( 8 galaxies and 4 stars) for which we measured radial velocities, but which are not contained in our final APM-derived catalog. These objects were measured during our first run, but because of their faintness (or imprecise coordinates), they have no matching object in our APM-derived catalog. For these objects we have estimated $r_{F}$ magnitudes using a fit between the PDS instrumental magnitudes and calibrated $\mathrm{r}_{F}$ magnitudes for galaxies presenting both measurements. None of the objects in Tables 4 and 5 will be considered further in the analyses that follow.

The number of galaxies with cross-correlation velocities is 155; those whose redshift was estimated from emission lines number 126, while 39 had both cross-correlation and emission line velocities. Thus, the proportion of objects presenting emission lines is $\sim 52 \%$, which is similar to that obtained by Bellanger et al. (1995) in their deeper survey ( $\mathrm{R} \leq$ 20.5) close to the south Galactic pole. The two quasars had been noted previously by 
Berger et al. (1991) as objects presenting ultra-violet excess.

In the analysis presented in paper I, in addition to the redshifts we measured, we also included in our catalog measurements from ZCAT (69 galaxies, some of which are unpublished, kindly provided by J. Huchra); van Haarlem et al. (1993, 3 velocities); Ellman (1994, 19 galaxies); Koo \& Kron (unpublished; 157 objects, of which 132 are galaxies) and one galaxy from Boroson, Salzer, \& Trotter (1993).

\subsection{Catalog Completeness}

Two different effects play a role in the redshift completeness. The first is the actual clustering of galaxies combined with the limitations imposed by the use of multi-object spectroscopy. For the mean density of objects at the limiting magnitude of this project $(\sim$ 300 galaxies per square degree), it is very difficult with the present technology to secure spectra for $100 \%$ of the objects in only one configuration. As mentioned above, the sample contains 1013 galaxies with $\mathrm{b}_{J} \leq 20$, of which a total of $375(37 \%)$ were assigned to fibers. This then, represents the completeness level imposed by the observational technique, though we should also mention that this number also depends on the number of configured fields (8), which was insufficient to cover the entire sample.

The second effect is the actual identification of features that would allow measuring a redshift of objects that were observed. Of the 375 observed galaxies to $b_{J}=20,273(73 \%)$ yielded a redshift, while 102 did not. An additional 165 objects fainter than the limiting magnitude were observed without producing a measurable spectrum. Thus, the efficiency, i.e., our ability to obtain a spectrum that allowed measuring a redshift, is $\sim 70 \%$ at the limiting magnitude of the survey, while, when the total number of positioned fibers is considered (i.e., including objects fainter than $\left.\mathrm{b}_{J}=20\right) \sim 59 \%$ yielded a redshift. 
The total number of galaxies with redshifts to $b_{J}=20$ is 383 , which includes in addition to our redshifts 110 measurements by other authors. The catalog completeness as a function of magnitude is presented in Figure 6(a) where the solid line represents the total fraction of galaxies with redshift measurements and the dotted line represents the proportion of galaxies contributed by this work. The overall completeness at the limiting magnitude is $\sim 35 \%$. This number is slightly lower than that quoted in paper I $(\sim 40 \%)$ because in that work we used a preliminary calibration which did not make any corrections of the APM isophotal magnitudes to obtain "total" magnitudes, nor did we take into account the dependence on color. When these corrections are used, in particular the size correction, the magnitudes of galaxies become "brighter" and so more objects are included in the sample.

\subsection{Astrometry errors}

We have investigated some of the possible causes for this rather low yield of redshifts for observed galaxies. The first we considered is the existence of astrometrical errors (in particular for the 1992 run). In Figures 7(a) and 7(b) we show the distribution of residuals in R.A. and decl. respectively, against R.A. from the match between the final combined catalog with that used in the first run. We can see that in general the residuals are less than $0.5^{\prime \prime}$, but become fairly dramatic in R.A. at the edges of the sample and in declination at an R.A. $\sim 194^{\circ}\left(12^{h} 56^{m}\right)$. From these figures, we conclude that poor coordinates could indeed be a likely cause for the low efficiency, particularly at the eastern edge of the sample, although we should note that this field was also the one presenting the smallest number of configured objects (65), owing to the lower projected surface density of objects. 


\subsection{Surface Brightness}

Another parameter we considered is the mean surface brightness. In Fig. 8 we present a histogram showing the distribution in surface brightness for galaxies observed successfully (solid line), while the dashed line represents galaxies whose spectra did not allow measuring a redshift. Here we can see a significant drop at an $\mathrm{SB} \sim 24.3 \mathrm{mag} \operatorname{arc~} \mathrm{sec}^{-2}$ for galaxies with measured redshifts. The median surface brightness of galaxies with measured redshifts (320) is $24.09 \pm 0.27$, compared to $24.39 \pm 0.30$ for objects with unknown redshifts (182). Although the difference between both values is only marginally larger than their estimated errors, the figure suggests that the surface brightness is a limiting factor in the successful measurement of spectra.

\subsection{Profile}

In Figure 9 we show a histogram of the profile slope, where, as above, galaxies with measurable spectra are represented by solid lines, while those which did not yield redshifts are represented by the dashed line. The profile slope was derived fitting an exponential power-law to the areal profiles of the APM catalog. The histogram suggests that the slope inclination could play a small role in the lack of measurable redshifts, in the sense that galaxies with flatter slopes are somewhat less likely to yield redshifts, though the difference between both distributions is really marginal.

\subsection{Color}

The galaxy color apparently is not an important parameter for the successful observation of objects, as can be seen in Figure 10. Although galaxies with redshifts peak at a bluer color, the median values for the distribution are essentially the same $\left(\mathrm{b}_{J}-\mathrm{r}_{F}\right)_{n o z}$ 
$=1.09 \pm 0.26$ and $\left(\mathrm{b}_{J}-\mathrm{r}_{F}\right)_{\text {with } z}=1.08 \pm 0.24$, respectively. As each of the observing runs used a catalog derived from a different color, we verified whether this could introduce a bias in the catalog of galaxies with redshifts (e.g., Ellman 1994). For the first run we find $\left(\mathrm{b}_{J}-\mathrm{r}_{F}\right)=1.08 \pm 0.25$, while for the second run it is $\left(\mathrm{b}_{J}-\mathrm{r}_{F}\right)=1.05 \pm 0.25$, which shows that there is no difference between the samples. The overall median color of the sample (i.e., all galaxies, with and without measured spectra) is very close to these values, being $\left(\mathrm{b}_{J}-\mathrm{r}_{F}\right)=1.02 \pm 0.25$. Finally, we inspected objects presenting very extreme colors. In general galaxies with very red colors either present saturation or have images classified as merged by APM, while most of the very blue objects are either faint, probably having large associated uncertainties, or have perturbed appearances.

\section{Conclusion}

We have presented a catalog containing 328 redshifts and magnitudes of galaxies observed in a $4^{\circ} \times 0.67^{\circ}$ minislice close to the direction of the north Galactic pole. A further 267 targets have been observed but did not produce measurable spectra. The completeness level of the catalog at its nominal limiting magnitude is $\sim 35 \%$. The incompleteness in our data is the result of a combination of several effects. The primary effect is the actual distribution of galaxies combined with the limitations imposed by the use of multiobject spectroscopy. Thus, part of the low redshift incompleteness is attributable to the insufficient number of configurations, as no field was observed twice in two different configurations, while the clustering of galaxies implies that some fields have more galaxies than others. Our efficiency rate in obtaining a redshift at the survey magnitude limit is $\sim$ $70 \%$.

We have shown in section 4 that other causes of incompleteness are present, e.g., poor coordinates might have played a role in the low efficiency in redshift acquisition, particularly 
for our easternmost field, but it is clearly not the only cause. In our data, the mean surface brightness of objects seems to be an important limiting factor, for which a break can be seen at $\sim \mathrm{SB}=24.3 \mathrm{~b}_{J}$ mag $\operatorname{arc~sec}^{-2}$. Neither the color nor the profile shape seems to play an important role in the ability of obtaining a measurable spectrum. We have shown that many of the objects in our sample presenting extreme colors have either saturated images in the J plate or were classified as merged objects by the APM software.

We would like to thank N. Reid for the loan of the Schmidt plates; the director of NOAO for the use of the PDS; Ed Carder for assistance during the PDS scans; the HYDRA team (S. Barden, T. Armandroff, P. Massey, and L. Groves) for their help related to the spectroscopy runs; A. Klemola for help in re-measuring the astrometry; D. Mink for providing RVSAO; S. Majewski for his catalog, and the anonymous referee whose comments have helped to improve the presentation of this paper. C.N.A.W. thanks S. Arnouts, L. da Costa, and V. de Lapparent for discussions. We acknowledge the use of the NASA Astrophysics Data System, the NASA National Space Science Data Center and the NED/IPAC Extragalactic Database, which is operated by the Jet Propulsion Laboratory, California Institute of Technology, under contract with the National Aeronautics and Space Administration. This work has been funded by the following grants: CNPq 201036/90.8; NSF AST-8858203, AST-9023178; the US-Hungarian Science and Technology Grant J.F. no. 010/90; California Space Institute CS 6-89 and a Sigma Xi grant. 
Table 1. Coordinate Residuals between Catalogs

\begin{tabular}{|c|c|c|c|c|c|c|c|c|}
\hline $\begin{array}{l}\text { Catalog } 1 \\
\text { (1) }\end{array}$ & $\begin{array}{c}\text { Catalog } 2 \\
(2)\end{array}$ & $\begin{array}{c}\mathrm{N}_{\mathrm{obj}} \\
(3)\end{array}$ & $\begin{array}{c}\Delta \alpha\left({ }^{\prime \prime}\right) \\
(4)\end{array}$ & $\begin{array}{l} \pm \\
(5)\end{array}$ & $\begin{array}{c}\Delta \delta\left(^{\prime \prime}\right) \\
(6)\end{array}$ & $\begin{array}{l} \pm \\
(7)\end{array}$ & $\begin{array}{c}\Delta p o s\left({ }^{\prime \prime}\right) \\
(8)\end{array}$ & $\begin{array}{l} \pm \\
(9)\end{array}$ \\
\hline GSC & $\mathrm{AME}$ & 442 & 0.96 & 0.33 & -0.03 & 0.22 & 0.91 & 0.38 \\
\hline PDS(1993) & PDS (1992) & 877 & 0.26 & 0.49 & 0.23 & 0.38 & 0.51 & 0.49 \\
\hline $\operatorname{APM}(\mathrm{J})$ & $\mathrm{APM}(\mathrm{F})$ & 25136 & 0.24 & 0.42 & 0.14 & 0.36 & 0.55 & 0.28 \\
\hline $\operatorname{APM}(J)$ & PDS (1993) & 7710 & 0.03 & 0.25 & 0.01 & 0.24 & 0.27 & 0.22 \\
\hline KK & $\operatorname{APM}(\mathrm{J})$ & 1436 & -0.57 & 0.53 & -0.59 & 0.52 & 1.02 & 0.43 \\
\hline KK & $\mathrm{APM}(\mathrm{F})$ & 1393 & -0.26 & 0.54 & -0.52 & 0.50 & 0.84 & 0.40 \\
\hline Majewski & $\operatorname{APM}(\mathrm{J})$ & 815 & 0.12 & 0.29 & -0.01 & 0.34 & 0.38 & 0.26 \\
\hline KRN & Final & 96 & 1.22 & 0.65 & -1.28 & 1.26 & 2.08 & 0.87 \\
\hline van Haarlem & Final & 19 & -0.25 & 0.95 & 0.29 & 0.73 & 0.93 & 0.82 \\
\hline
\end{tabular}

Note. - Cols. (1) and (2) identification of catalog: GSC, Lasker et al. (1990); AME, remeasurement of GSC objects using Lick Astrograph plate; PDS, catalog derived from Focas scans, with coordinates derived from the Guide Star Catalog (PDS 1992) or from Lick Astrograph plate (PDS 1993); APM, Catalogs derived from APM scans of J and F plates; KK, coordinates from the catalog of D.C. Koo \& R.G. Kron (private communication) for SA 57; Majewski, Catalog of S.R. Majewski (private communication) for SA 57; KRN, Kent et al. 1993; van Haarlem: van Haarlem et al. 1993; Final, final catalog of objects prepared for this work. Col. (3) number of objects in common. Col. (4) mean difference in the sense catalog 1 - catalog 2 for right Ascension, Col. (5) its associated uncertainty. Col. (6) mean difference in declination (catalog 1 - catalog 2 ) and Col. (7) uncertainty. Col. (8) mean quadratic difference between positions, combining the differences in right ascension and declination and Col.(9) its associated uncertainty. 
Table 2. Radial Velocities in common with other Sources

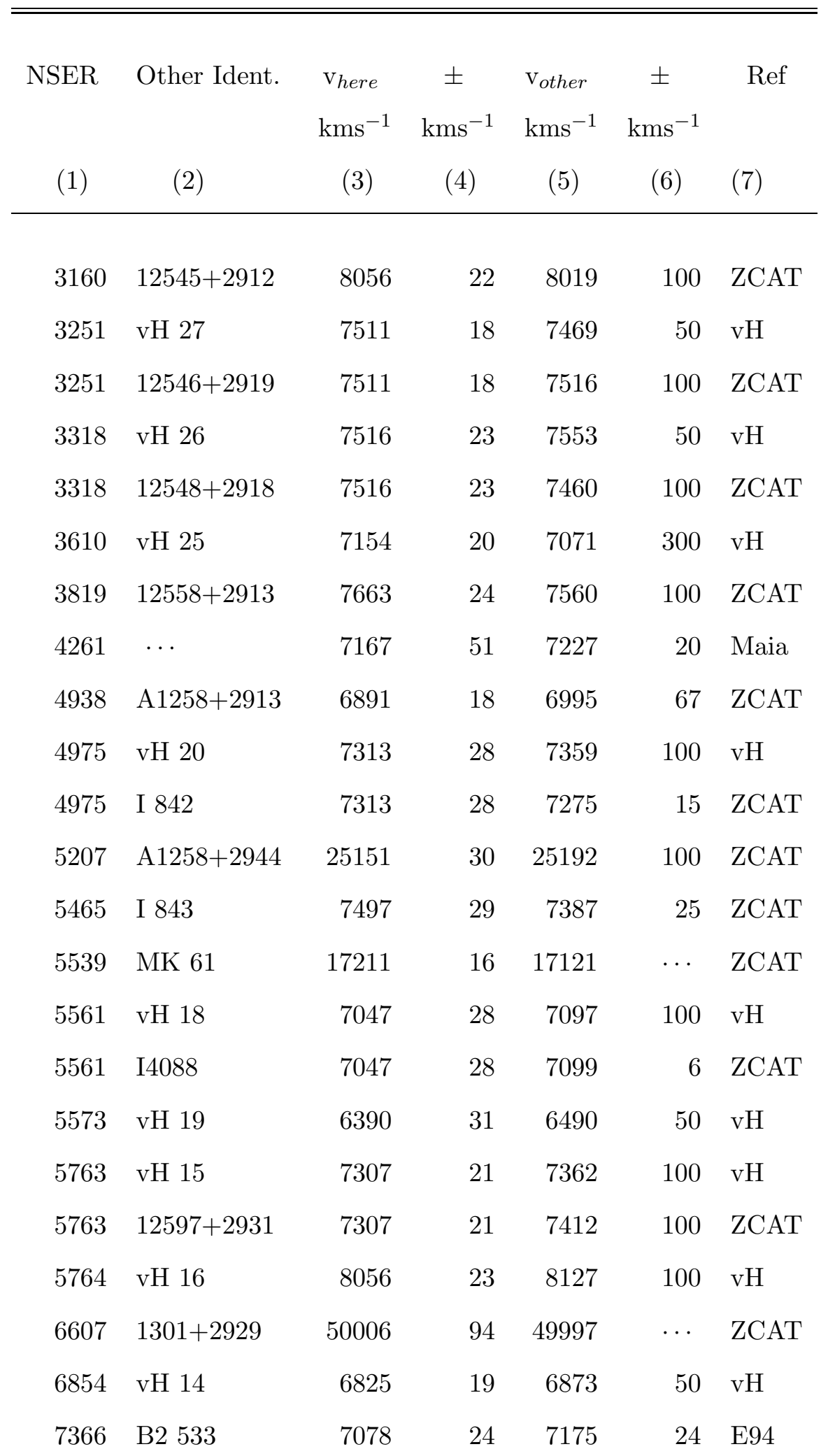


Table 2-Continued

\begin{tabular}{|c|c|c|c|c|c|c|}
\hline NSER & Other Ident. & & & & & Ref \\
\hline$(1)$ & (2) & $\begin{array}{c}\mathrm{kms}^{-1} \\
(3)\end{array}$ & $\begin{array}{c}\mathrm{kms}^{-1} \\
(4)\end{array}$ & $\begin{array}{c}\mathrm{kms}^{-1} \\
(5)\end{array}$ & $\begin{array}{c}\mathrm{kms}^{-1} \\
(6)\end{array}$ & (7) \\
\hline 7366 & $13030+2934$ & 7078 & 24 & 7158 & 100 & ZCAT \\
\hline 7646 & B2 513 & 15271 & 8 & 15353 & 22 & E94 \\
\hline 7766 & B2 521 & 7389 & 11 & 7532 & 62 & E94 \\
\hline 7915 & B2 512 & 25256 & 11 & 25362 & 96 & E94 \\
\hline 8081 & B2 509 & 54491 & 9 & 54675 & $\ldots$ & E94 \\
\hline 9848 & N 5004A & 7217 & 28 & 7262 & 20 & ZCAT \\
\hline$\cdots$ & N 4472 & 931 & 21 & 997 & 10 & ZCAT \\
\hline$\cdots$ & HD 66141 & 53 & 5 & 71 & 1 & $\mathrm{AA}$ \\
\hline$\cdots$ & HD 107328 & 35 & 22 & 36 & 1 & $\mathrm{AA}$ \\
\hline$\cdots$ & HD 123782 & -26 & 14 & -13 & 1 & $\mathrm{AA}$ \\
\hline$\cdots$ & HD 132737 & -15 & 2 & -24 & 1 & $\mathrm{AA}$ \\
\hline$\cdots$ & HD 132737 & -49 & 4 & -24 & 1 & $\mathrm{AA}$ \\
\hline
\end{tabular}

Note. - Col. (1) serial number in our main catalog. Col. (2) other identification. Col.(3) radial velocity measured in this work and in Col. (4) its associated internal uncertainty. Col. (5) radial velocity, Col. (6), uncertainty and Col. (7) reference of other measurements.

References. - Blumberg \& Boksenberg (1995, AA); Ellman (1994, E94); M.A.G. Maia (private communication, Maia) ; van Haarlem et al. (1993, vH); Huchra (Private Communication, ZCAT) 
Table 4. Redshifts and Magnitudes for Observed Stars

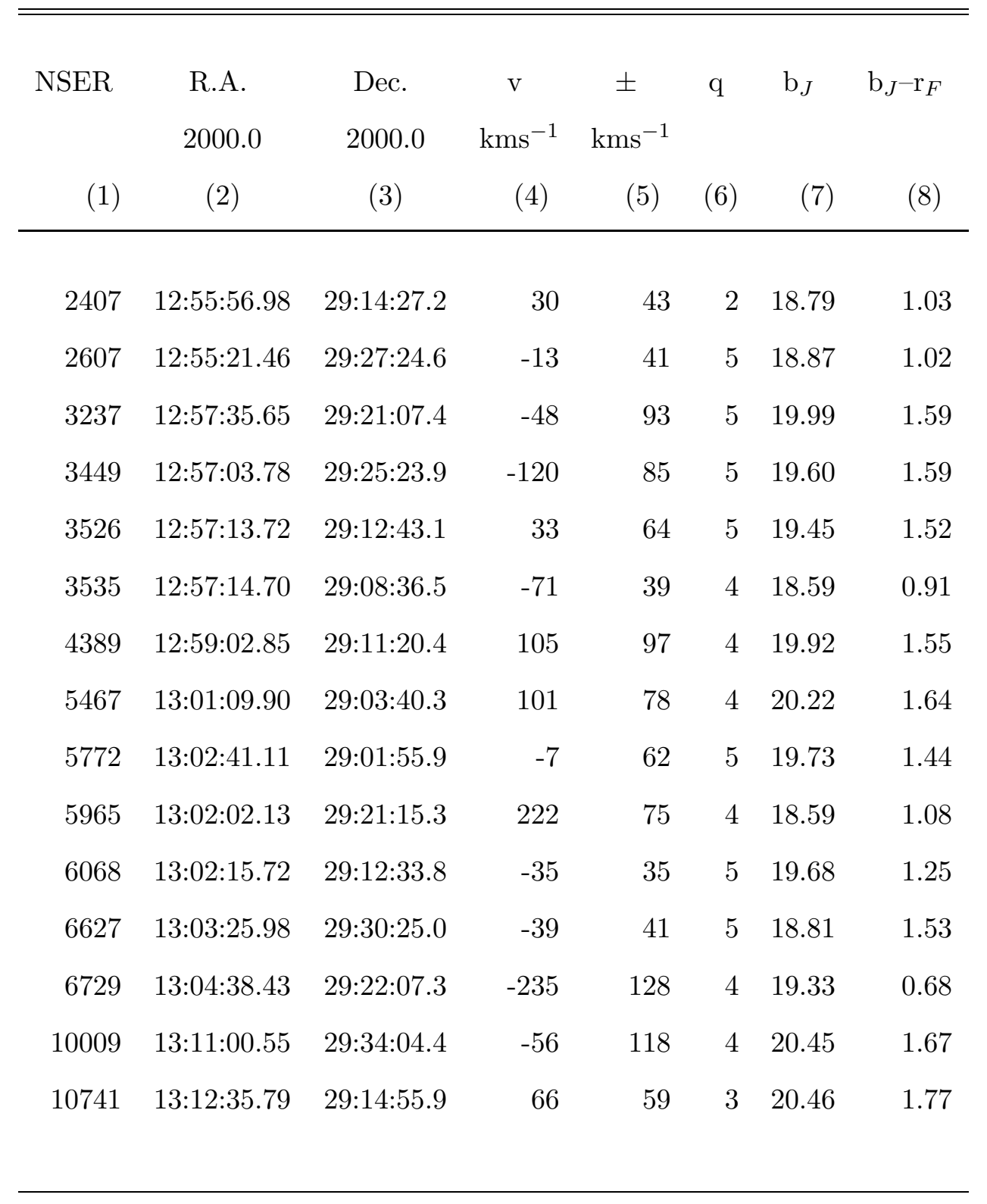

Note. - Col. (1) serial number in our main catalog. Col. (2) right ascension. Col. (3) declination. Col. (4) radial velocity. Col. (5) internal error of radial velocity. Col. (6) redshift quality derived from the number of identified features in spectrum. Col. (7) magnitude. Col. (8) color. 
Table 5. Redshifts of Objects not in the Main Catalog

\begin{tabular}{cccccccc}
\hline \hline & & & & & & & \\
PDS Id. & R.A. & Dec. & $\mathrm{z}$ & t & $\mathrm{t}$ & $\mathrm{q}$ & estimated $\mathrm{r}_{F}$ \\
& 2000.0 & 2000.0 & & $\mathrm{kms}^{-1}$ & & & \\
$(1)$ & $(2)$ & $(3)$ & $(4)$ & $(5)$ & $(6)$ & $(7)$ & $(8)$ \\
\hline 882 & $12: 55: 50.71$ & $29: 11: 22.49$ & 0.19788 & 54 & $\mathrm{x}$ & 1 & 19.84 \\
686 & $12: 57: 14.41$ & $29: 12: 24.55$ & 0.35489 & 36 & $\mathrm{e}$ & 5 & 19.23 \\
698 & $12: 58: 46.52$ & $28: 58: 26.20$ & -0.00062 & 237 & $\mathrm{x}$ & 3 & 19.01 \\
889 & $12: 59: 02.57$ & $29: 14: 22.19$ & -0.00050 & 107 & $\mathrm{x}$ & 5 & 19.51 \\
704 & $13: 01: 16.24$ & $29: 34: 03.58$ & 0.32904 & 105 & $\mathrm{x}$ & 3 & 19.29 \\
638 & $13: 02: 46.72$ & $29: 11: 47.81$ & 0.39613 & 62 & $\mathrm{c}$ & 4 & 19.13 \\
655 & $13: 03: 57.29$ & $29: 13: 48.69$ & 0.38763 & 104 & $\mathrm{x}$ & 2 & 19.15 \\
959 & $13: 04: 04.18$ & $29: 16: 22.42$ & -0.00014 & 44 & $\mathrm{x}$ & 3 & 19.89 \\
630 & $13: 04: 48.69$ & $29: 10: 34.71$ & 0.38778 & 143 & $\mathrm{x}$ & 2 & 19.11 \\
988 & $13: 11: 06.28$ & $29: 14: 38.22$ & 0.00008 & 125 & $\mathrm{x}$ & 2 & 20.33 \\
978 & $13: 11: 32.38$ & $29: 21: 35.50$ & 0.23847 & 95 & $\mathrm{x}$ & 1 & 20.64 \\
858 & $13: 12: 46.08$ & $28: 58: 13.23$ & 0.32588 & 60 & $\mathrm{x}$ & 1 & 19.74 \\
& & & & & & & \\
\hline
\end{tabular}

Note. - Col. (1) serial number in catalog derived from PDS scans used in the first observing run. Col. (2) right ascension. Col. (3) declination. Col. (4) observed redshift. Col. (5) internal radial velocity error. Col. (6) type of redshift: crosscorrelation (x), emission (e) or combined cross-correlation and emission (c). Col. (7) redshift quality derived from the number of identified features in the spectrum. Col. (8) $r_{F}$ magnitude estimated from a fit between the instrumental magnitude measured with the PDS microdensitometer calibrated $\mathrm{r}_{F}$ magnitudes. The latter have uncertainties of $\sim 0.20 \mathrm{mag}$ 


\section{REFERENCES}

Barden, S.C., Armandroff, T., Massey, P., Groves, L., Rudeen, A.C., Vaughnn, D. \& Muller, G. 1992, ASP Conf. Ser. Vol. 37, "Fiber Optics in Astronomy", ed. Peter M. Gray (San Francisco: ASP), 185

Bellanger, C., de Lapparent, V., Arnouts, S., Mathez, G., Mazure, A., \& Mellier, Y. 1995, A\&AS, 110, 159

Berger, J., Cordoni, J. P., Fringant, A. M., Guibert, J., Moreau, O., Reboul, H., and Vanderriest, C. 1991, A\&AS, 87, 389

Bershady, M.A., Hereld, M., Kron, R.G., Koo, D.C., Munn, J.A., \& Majewski, S.R. 1994, AJ, 108,870

Blumberg, R.E., \& Boksenberg, A., ed. 1995, "The Astronomical Almanac for the Year 1995”, (Washington: U.S. Government Printing Office)

Boroson, T.A., Salzer, J.J., Trotter,A. 1993, ApJ, 412, 524

Broadhurst, T.J., Ellis, R.S., Koo, D.C., \& Szalay, A.S. 1990, Nature, 343, 726

Corbin,T., Urban,S. 1988, in I.A.U. Symp. No. 133, "Mapping the Sky", ed. H. Eichhorn (Dordrecht: Reidel), 75

da Costa, L.N., et al. 1988, AJ, 327, 544

da Costa, L.N., et al. 1989, AJ, 97, 315

de Lapparent, V., Geller, M.J., \& Huchra, J.P, 1991, ApJ, 369, 273

Ellman, N. 1994, Ph.D. thesis, Univ. California, Santa Cruz

Geller, M.J., \& Huchra, J.P. 1989, Science, 246, 897

Huchra, J.P. 1993, private communication (ZCAT)

Infante, L., \& Pritchet, C.J., 1992, ApJS, 83, 237 
Jacoby, G.H., Hunter, D.A., \& Christian, C.A. 1984, ApJS, 56, 257

Jarvis, J.F., \& Tyson, J.A. 1981, AJ, 86, 476

Kent, S.M., Ramella, M., \& Nonino, M. 1993, AJ, 105, 393 (KRN)

Kibblewhite, E.J., Bridgeland, M.T., Bunclark, P. \& Irwin, M.J. 1984, in Astronomical Microdensitometry Conference, ed. D.A. Klinglesmith (NASA CP 2317), 277

Klemola, A.R., Jones, B.F., Hanson, R.B. 1987, AJ, 94, 501

Koo, D.C. 1986, ApJ, 311, 651

Koo, D.C., Ellman, N., Kron, R.G., Munn, J.A., Szalay, A.S., Broadhurst, T.J., \& Ellis, R.S. 1993, in ASP Conf. Ser. Vol. 51, Observational Cosmology Symposium, ed. G. Chincarini, A. Iovino, T. Maccacaro, \& D. Maccagni (San Francisco: ASP), 112

Kron, R.G. 1980, ApJS, 43, 305

Kurtz, M.J., Mink, D.J.,Wyatt, W.F., Fabricant, D.G., Torres, G., Kriss, G.A., \& Tonry, J.L. 1992, in ASP Conf. Ser. Vol. 25, Proc. $1^{\text {st }}$ Ann. Conf. Astronomical Data Analysis Software and Systems, ed., D.M. Worral, C. Biemesderfer, \& J. Barnes (San Francisco: ASP), 432

Lasker, B.M., Sturch, C.R., McLean, B.M., Russel, J.L., Jenker, H., \& Shara, M. 1990, AJ, 99, 2019 (GSC)

Maddox, S.J., Sutherland, W.J., Efstathiou, G., \& Loveday, J. 1990, MNRAS, 243, 692

Massey, P. 1992, A User's Guide to CCD Reductions with IRAF (Tucson: KPNO Computer Support Group)

Ramella, M., Geller, M.J., \& Huchra, J.P. 1992, ApJ, 384, 396

Reid, I. N., Brewer, C., McKinley, W., \& Maurey, A. 1991, PASP, 103, 661

Röser, S., \& Bastian, U. 1991, PPM Star Catalogue (Heidelberg: Spektrum Akademischer Verlag) 
Tonry, J., \& Davis, M. 1979, AJ, 84, 1511

Valdes, F. 1982, Focas User's Manual (2 $2^{\text {nd }}$ ed.; Tucson: KPNO Computer Support Group)

van Haarlem, M.P., Cayón, L., de la Cruz, G.G., Martínez-González, E. \& Rebolo, R. 1993, MNRAS, 264, 71

Willmer, C. N. A., Koo, D. C., Szalay, A. S., \& Kurtz, M. J. 1994, ApJ, 437, 560 (Paper I)

This manuscript was prepared with the AAS LATEX macros v4.0. 
Fig. 1.- Residuals between Lick Astrograph and GSC coordinates of GSC stars within the boundaries of this survey. The length of the arrow at the top left corner corresponds to 1 arc second. The systematic difference between coordinates of both solutions is immediately apparent.

Fig. 2.- Comparison between magnitudes calculated using the transformations described in section 2.2 compared to the Koo and Kron catalog. Filled circles represent galaxies used in the calibration, while those that were not used are shown as open triangles. These comparisons are shown as (a) a difference in magnitudes in $b_{J}$, and (b) differences in color against $\mathrm{b}_{J}$.

Fig. 3.- Distribution in magnitudes of galaxies with pixels above emulsion non-linearity in each band. These are represented by short dashed lines in (a) and (b), while the total number of galaxies is shown as a solid histogram.

Fig. 4. - Examples of galaxy spectra measured for this work. (a e) Typical examples of galaxies for which we were successful in measuring cross-correlation redshifts; these figures are ordered in decreasing redshift quality, which is related to the number of features that can be identified in the spectra. (f $\sim \mathrm{j})$ Spectra of galaxies for which we secured emission-line redshifts. At the top left corner we show the galaxy identification, redshift and its quality from Table 3, except in the case of (e), where the data are taken from Table 5. Under the spectra, the expected positions of some prominent absorption lines are noted. Emission lines are identified above the spectra. 
Fig. 5.- Distribution of signal-to-noise ratio against internal radial velocity uncertainty. Filled squares represent galaxies with both emission and cross-correlation velocities; open squares stand for galaxies with cross-corrrelation only, and crosses represent those with emission only. The ability of measuring radial velocities at low signal-to-noise levels for objects with emission lines is immediately apparent. In general, the smallest signal-to-noise ratio for which cross-correlation velocities can be measured is about 3, while above 9 all objects have measured cross-correlations.

Fig. 6.- Redshift completeness as a function of apparent magnitude, for the whole sample of galaxies. The dashed line represents the fraction of galaxies that were contributed by this work.

Fig. 7.- Residuals of coordinate differences between the final catalog and the catalog in the first observing run plotted as function of (a) right ascension and (b) declination. This plot suggests that poor coordinates could have been a cause of the rather low detection rate of objects during the first observing run.

Fig. 8.- Histogram showing the mean surface brightness distribution for galaxies with redshifts (plotted as solid lines) and for galaxies that were observed, but for which no features could be identified in the spectrum (dashed line). There is a significant break in the success rate for galaxies fainter than about $23.4 \mathrm{mag}$ arc $\mathrm{sec}^{-2}$.

Fig. 9.- Distribution of profile slope measured in the J plate for galaxies with measured redshifts (solid line) and unsuccessfully observed (dashed line). Both distributions are similar, suggesting that the profile shape is not an important parameter in determining whether a redshift will be successfully obtained. 
Fig. 10.- Color distribution of galaxies with measured redshifts (solid line) and without (dashed line). This diagram suggests that our ability of obtaining a measurable spectrum in not correlated with the galaxy color. 
TABLE 3

Redshifts, Magnitudes and Colors for Observed Galaxies

\begin{tabular}{|c|c|c|c|c|c|c|c|c|c|c|c|}
\hline $\begin{array}{r}\text { NSER } \\
\text { (1) }\end{array}$ & $\begin{array}{l}\text { R.A. } \\
2000.0 \\
(2)\end{array}$ & $\begin{array}{l}\text { Dec. } \\
2000.0 \\
(3)\end{array}$ & $(4)$ & $\begin{array}{c} \pm \\
\mathrm{kms}^{-1} \\
(5)\end{array}$ & (6) & (7) & $\begin{array}{l}\mathrm{b}_{J} \\
(8)\end{array}$ & $\begin{array}{c}\mathrm{b}_{J}-\mathrm{r}_{F} \\
(9)\end{array}$ & $\begin{array}{c}\operatorname{sat}\left(\mathrm{b}_{J}\right) \\
\% \\
(10)\end{array}$ & $\begin{array}{c}\operatorname{sat}\left(\mathrm{r}_{F}\right) \\
\% \\
(11)\end{array}$ & $\begin{array}{c}\mathrm{SB} \\
\mathrm{b}_{J} \operatorname{arcsec}^{-2} \\
(12)\end{array}$ \\
\hline 2103 & $12: 54: 45.81$ & $29: 04: 42.4$ & 0.1966 & 24 & $\mathrm{e}$ & 3 & 20.26 & 0.80 & 0 & 0 & 23.90 \\
\hline 2110 & $12: 54: 46.58$ & $29: 00: 54.6$ & 0.2552 & 115 & $\mathrm{x}$ & 3 & 19.47 & 1.18 & 0 & 0 & 24.15 \\
\hline 2130 & $12: 54: 48.52$ & $29: 13: 27.1$ & 0.1422 & 35 & $\mathrm{e}$ & 3 & 19.85 & 0.94 & 0 & 0 & 24.18 \\
\hline 2163 & $12: 54: 52.42$ & $29: 30: 05.6$ & 0.1837 & 121 & $\mathrm{x}$ & 4 & 19.83 & 1.36 & 0 & 0 & 24.20 \\
\hline 2183 & $12: 54: 55.32$ & $29: 00: 43.7$ & 0.0214 & 55 & $\mathrm{x}$ & 5 & 17.46 & 1.06 & 1 & 0 & 24.19 \\
\hline 2213 & $12: 54: 58.99$ & $29: 16: 06.4$ & 0.0620 & 19 & $\mathrm{e}$ & 4 & 19.26 & 0.75 & 0 & 0 & 23.67 \\
\hline 2262 & $12: 55: 05.25$ & $29: 16: 39.8$ & 0.2596 & 17 & $\mathrm{e}$ & 5 & 19.56 & 0.86 & 0 & 0 & 24.12 \\
\hline 2335 & $12: 55: 13.66$ & 29:08:56.1 & 0.1979 & 27 & $\mathrm{e}$ & 3 & 19.38 & 0.98 & 0 & 0 & 24.09 \\
\hline 2345 & $12: 55: 15.33$ & $28: 58: 53.4$ & 0.1979 & 22 & $\mathrm{e}$ & 5 & 19.04 & 1.18 & 0 & 0 & 24.06 \\
\hline 2347 & $12: 55: 15.37$ & $29: 01: 51.5$ & 0.1421 & 65 & $\mathrm{x}$ & 4 & 19.74 & 1.29 & 0 & 0 & 24.11 \\
\hline 2359 & $12: 55: 16.71$ & $29: 28: 54.3$ & 0.2323 & 127 & $\mathrm{x}$ & 3 & 20.33 & 1.59 & 0 & 0 & 24.41 \\
\hline 2437 & $12: 55: 26.06$ & $28: 57: 28.2$ & 0.2380 & 162 & $\mathrm{x}$ & 2 & 19.24 & 0.97 & 0 & 0 & 24.28 \\
\hline 2470 & $12: 55: 29.46$ & $29: 29: 24.8$ & 0.0677 & 18 & $\mathrm{e}$ & 4 & 18.87 & $\ldots$ & 0 & $\ldots$ & 24.87 \\
\hline 2482 & $12: 55: 30.61$ & $29: 05: 41.3$ & 0.1984 & 37 & $\mathrm{c}$ & 4 & 19.56 & 1.05 & 0 & 0 & 24.06 \\
\hline 2510 & $12: 55: 34.85$ & 29:00:02.4 & 0.0624 & 43 & $\mathrm{x}$ & 4 & 17.20 & 1.10 & 1 & 0 & 24.19 \\
\hline 2581 & $12: 55: 42.77$ & $29: 25: 28.5$ & 0.2771 & 24 & $\mathrm{e}$ & 2 & 20.33 & 0.89 & 0 & 0 & 24.13 \\
\hline 2586 & $12: 55: 43.21$ & $29: 27: 39.1$ & 0.1891 & 62 & $\mathrm{e}$ & 4 & 19.76 & 1.04 & 0 & 0 & 24.07 \\
\hline 2627 & $12: 55: 49.27$ & $29: 03: 54.4$ & 0.2522 & 56 & $\mathrm{x}$ & 5 & 19.52 & 1.60 & 0 & 0 & 23.89 \\
\hline 2668 & $12: 55: 53.33$ & $29: 03: 56.4$ & 0.0329 & 21 & $\mathrm{e}$ & 5 & 18.95 & 0.46 & 0 & 0 & 24.70 \\
\hline 2696 & $12: 55: 56.27$ & $29: 02: 59.0$ & 0.1604 & 86 & $\mathrm{x}$ & 4 & 20.46 & 1.43 & 0 & 0 & 24.15 \\
\hline 2704 & $12: 55: 57.42$ & $28: 55: 55.6$ & 0.1967 & 34 & $\mathrm{e}$ & 4 & 19.74 & 1.18 & 0 & 0 & 24.02 \\
\hline 2722 & $12: 55: 59.18$ & $29: 22: 53.4$ & 0.1640 & 21 & $\mathrm{e}$ & 4 & 18.96 & 1.01 & 0 & 0 & 23.67 \\
\hline 2749 & $12: 56: 01.63$ & $29: 15: 11.2$ & 0.0680 & 28 & $\mathrm{c}$ & 5 & 16.77 & 0.96 & 5 & 1 & 23.49 \\
\hline 2752 & $12: 56: 01.90$ & $29: 15: 48.3$ & 0.0678 & 18 & $\mathrm{e}$ & 5 & 18.06 & 0.81 & 0 & 0 & 24.08 \\
\hline 2753 & $12: 56: 02.00$ & $29: 12: 13.0$ & 0.1964 & 68 & $\mathrm{x}$ & 4 & 19.84 & 1.44 & 0 & 0 & 24.48 \\
\hline 2767 & $12: 56: 03.68$ & 29:09:23.4 & 0.1949 & 22 & $\mathrm{e}$ & 2 & 20.82 & 1.17 & 0 & 0 & 24.54 \\
\hline 2782 & $12: 56: 05.31$ & $29: 06: 13.4$ & 0.2367 & 56 & $\mathrm{x}$ & 3 & 20.26 & 1.43 & 0 & 0 & 24.09 \\
\hline 2791 & $12: 56: 06.51$ & $29: 17: 10.8$ & 0.0908 & 23 & $\mathrm{e}$ & 5 & 18.58 & 0.70 & 1 & 0 & 23.66 \\
\hline 2832 & $12: 56: 11.50$ & $29: 23: 52.8$ & 0.0240 & 21 & $\mathrm{e}$ & 5 & 18.95 & 0.88 & 0 & 0 & 23.97 \\
\hline 2833 & $12: 56: 11.80$ & $28: 55: 00.6$ & 0.0235 & 28 & $\mathrm{e}$ & 4 & 19.14 & 0.67 & 0 & 0 & 23.81 \\
\hline 2872 & $12: 56: 16.36$ & $28: 57: 42.5$ & 0.1983 & 107 & $\mathrm{x}$ & 4 & 20.20 & 1.46 & 0 & 0 & 24.28 \\
\hline 2897 & $12: 56: 18.88$ & $29: 13: 19.2$ & 0.1949 & 29 & $\mathrm{e}$ & 4 & 19.94 & 0.88 & 0 & 0 & 23.96 \\
\hline 2910 & $12: 56: 20.22$ & $29: 17: 59.8$ & 0.0223 & 28 & $\mathrm{x}$ & 4 & 16.88 & 0.86 & 2 & 0 & 24.29 \\
\hline 2939 & $12: 56: 23.63$ & 29:00:30.9 & 0.1883 & 22 & $\mathrm{e}$ & 3 & 19.86 & 0.96 & 0 & 0 & 24.16 \\
\hline 2986 & $12: 56: 27.77$ & $29: 05: 46.6$ & 0.1631 & 61 & $\mathrm{x}$ & 5 & 18.89 & 1.30 & 0 & 0 & 23.89 \\
\hline 2992 & $12: 56: 28.63$ & $29: 08: 12.8$ & 0.0317 & 31 & $\mathrm{c}$ & 5 & 17.90 & 0.62 & 2 & 0 & 23.87 \\
\hline 3003 & $12: 56: 29.74$ & $29: 09: 45.1$ & 0.0660 & 26 & $\mathrm{e}$ & 3 & 19.34 & 0.31 & 0 & 0 & 24.58 \\
\hline 3033 & $12: 56: 33.22$ & $29: 11: 43.4$ & 0.2298 & 38 & $\mathrm{c}$ & 5 & 19.41 & 0.93 & 0 & 0 & 24.08 \\
\hline 3057 & $12: 56: 35.51$ & $29: 20: 27.7$ & 0.2654 & 52 & $\mathrm{x}$ & 3 & 20.22 & 1.52 & 0 & 0 & 24.38 \\
\hline 3060 & $12: 56: 35.92$ & $28: 56: 50.8$ & 0.0614 & 19 & $\mathrm{e}$ & 5 & 17.57 & 0.90 & 2 & 0 & 23.60 \\
\hline 3080 & $12: 56: 38.58$ & $29: 10: 18.4$ & 0.1735 & 50 & $\mathrm{x}$ & 4 & 20.14 & 1.45 & 0 & 0 & 23.90 \\
\hline 3097 & $12: 56: 41.53$ & $29: 22: 40.1$ & 0.2913 & 70 & $\mathrm{x}$ & 5 & 20.30 & 1.71 & 0 & 0 & 23.97 \\
\hline 3110 & $12: 56: 43.54$ & $29: 22: 27.2$ & 0.2937 & 102 & $\mathrm{x}$ & 2 & 19.63 & 1.35 & 0 & 0 & 24.00 \\
\hline 3160 & $12: 56: 50.55$ & $28: 55: 46.1$ & 0.0269 & 22 & $\mathrm{x}$ & 5 & 16.33 & 1.02 & 4 & 1 & 23.85 \\
\hline 3170 & $12: 56: 51.15$ & $29: 22: 40.8$ & 0.0239 & 24 & $\mathrm{x}$ & 5 & 16.94 & 0.98 & 2 & 1 & 24.05 \\
\hline 3192 & $12: 56: 54.85$ & $28: 57: 41.1$ & 0.0671 & 67 & $\mathrm{x}$ & 5 & 18.72 & 1.24 & 0 & 0 & 24.14 \\
\hline 3194 & $12: 56: 55.47$ & $28: 57: 21.6$ & 0.0681 & 35 & $\mathrm{x}$ & 5 & 17.69 & 1.27 & 2 & 1 & 23.91 \\
\hline 3208 & $12: 56: 56.71$ & $29: 03: 18.7$ & 0.1868 & 35 & $\mathrm{c}$ & 5 & 18.91 & 1.01 & 0 & 0 & 24.15 \\
\hline 3251 & $12: 57: 01.53$ & $29: 03: 44.3$ & 0.0250 & 18 & $\mathrm{x}$ & 5 & 16.58 & 1.32 & 6 & 2 & 23.63 \\
\hline 3257 & $12: 57: 02.13$ & $29: 24: 52.2$ & 0.1870 & 21 & $\mathrm{e}$ & 5 & 19.45 & 0.67 & 0 & 0 & 23.95 \\
\hline 3318 & $12: 57: 11.34$ & $29: 02: 41.5$ & 0.0251 & 23 & $\mathrm{c}$ & 5 & 16.11 & 1.32 & 5 & 2 & 23.74 \\
\hline 3341 & $12: 57: 14.08$ & $29: 28: 46.4$ & 0.1866 & 22 & $\mathrm{e}$ & 4 & 19.51 & 0.63 & 0 & 0 & 24.45 \\
\hline 3441 & $12: 57: 27.20$ & $29: 06: 20.6$ & 0.0613 & 26 & $\mathrm{e}$ & 2 & 19.63 & 0.74 & 0 & 0 & 24.09 \\
\hline 3467 & $12: 57: 30.07$ & $28: 57: 33.2$ & 0.2665 & 29 & $\mathrm{e}$ & 4 & 19.74 & 1.07 & 0 & 0 & 24.03 \\
\hline 3491 & $12: 57: 34.31$ & $28: 56: 11.5$ & 0.1495 & 109 & $\mathrm{x}$ & 4 & 20.33 & 1.40 & 0 & 0 & 24.18 \\
\hline 3540 & $12: 57: 39.79$ & $29: 29: 46.7$ & 0.1643 & 86 & $\mathrm{x}$ & 5 & 19.99 & 1.52 & 0 & 0 & 24.42 \\
\hline 3574 & $12: 57: 43.38$ & $28: 55: 53.0$ & 0.2618 & 68 & $\mathrm{x}$ & 4 & 20.14 & 1.70 & 0 & 0 & 24.27 \\
\hline 3610 & $12: 57: 46.98$ & $29: 08: 57.6$ & 0.0239 & 20 & $\mathrm{x}$ & 5 & 16.69 & 1.09 & 3 & 1 & 23.99 \\
\hline 3631 & $12: 57: 49.40$ & $29: 02: 42.1$ & 0.0230 & 25 & $\mathrm{e}$ & 5 & 19.14 & 0.45 & 0 & 0 & 23.92 \\
\hline 3639 & $12: 57: 50.80$ & $29: 12: 08.5$ & 0.1983 & 138 & $\mathrm{x}$ & 5 & 20.72 & 1.55 & 0 & 0 & 24.90 \\
\hline 3704 & $12: 58: 00.28$ & $29: 12: 20.4$ & 0.2985 & 96 & $\mathrm{e}$ & 2 & 20.11 & 1.19 & 0 & 0 & 24.17 \\
\hline 3738 & $12: 58: 03.92$ & $29: 20: 52.9$ & 0.0904 & 17 & e & 5 & 18.48 & 0.79 & 0 & 0 & 23.58 \\
\hline 3752 & $12: 58: 05.55$ & $29: 01: 02.2$ & 0.0259 & 43 & $\mathrm{x}$ & 5 & 18.23 & 0.97 & 0 & 0 & 24.74 \\
\hline 3766 & $12: 58: 06.79$ & $29: 02: 03.7$ & 0.0247 & 65 & $\mathrm{x}$ & 5 & 17.99 & 0.78 & 0 & 0 & 24.71 \\
\hline 3819 & $12: 58: 13.27$ & $28: 56: 53.0$ & 0.0256 & 24 & $\mathrm{x}$ & 5 & 16.59 & 1.23 & 4 & 2 & 23.83 \\
\hline 3828 & $12: 58: 14.84$ & $29: 14: 34.4$ & 0.0880 & 27 & $\mathrm{e}$ & 5 & 18.53 & 0.81 & 0 & 0 & 23.83 \\
\hline 3840 & $12: 58: 16.89$ & $29: 07: 06.7$ & 0.0613 & 30 & $\mathrm{e}$ & 3 & 19.30 & 0.43 & 0 & 0 & 24.26 \\
\hline 3861 & $12: 58: 20.08$ & $29: 18: 10.9$ & 0.2666 & 17 & e & 1 & 20.92 & 1.24 & 0 & 0 & 25.16 \\
\hline 3869 & $12: 58: 20.94$ & $29: 10: 54.2$ & 0.0893 & 79 & $\mathrm{x}$ & 2 & 18.67 & 0.68 & 0 & 0 & 24.40 \\
\hline 3886 & $12: 58: 23.93$ & $29: 06: 53.8$ & 0.0311 & 23 & $\mathrm{e}$ & 5 & 19.62 & 0.94 & 0 & 0 & 23.95 \\
\hline
\end{tabular}


TABLE 3-Continued

\begin{tabular}{|c|c|c|c|c|c|c|c|c|c|c|c|}
\hline NSER & $\begin{array}{l}\text { R.A. } \\
2000.0 \\
\quad(2)\end{array}$ & $\begin{array}{l}\text { Dec. } \\
2000.0 \\
(3)\end{array}$ & (4) & $\begin{array}{c} \pm \\
\mathrm{kms}_{(5)}^{-1}\end{array}$ & (6) & $\begin{array}{l}\mathrm{q} \\
(7)\end{array}$ & $\begin{array}{l}\mathrm{b}_{J} \\
(8)\end{array}$ & $\begin{array}{c}\mathrm{b}_{J}-\mathrm{r}_{F} \\
(9)\end{array}$ & $\begin{array}{c}\operatorname{sat}\left(\mathrm{b}_{J}\right) \\
\% \\
(10)\end{array}$ & $\begin{array}{c}\operatorname{sat}\left(\mathrm{r}_{F}\right) \\
\% \\
(11)\end{array}$ & $\begin{array}{c}\mathrm{SB} \\
\mathrm{b}_{J} \underset{(12)}{\operatorname{arcsec}} \\
(12\end{array}$ \\
\hline 4021 & $12: 58: 39.08$ & 29:00:52.8 & 0.1644 & 84 & $\mathrm{x}$ & 5 & 19.36 & 1.38 & 0 & 0 & 24.14 \\
\hline 4051 & $12: 58: 43.06$ & $29: 00: 09.3$ & 0.0851 & 27 & $\mathrm{e}$ & 3 & 19.95 & $\ldots$ & 0 & $\ldots$ & 24.18 \\
\hline 4052 & $12: 58: 43.15$ & $28: 54: 35.6$ & 0.0277 & 29 & $\mathrm{c}$ & 5 & 16.42 & 0.89 & 2 & 1 & 23.79 \\
\hline 4161 & $12: 58: 57.20$ & $29: 13: 23.2$ & 0.2317 & 58 & $\mathrm{x}$ & 5 & 19.57 & 1.20 & 0 & 0 & 24.43 \\
\hline 4189 & 12:59:00.24 & 29:11:00.9 & 0.0610 & 23 & $\mathrm{x}$ & 6 & 17.81 & 1.14 & 1 & 1 & 23.77 \\
\hline 4190 & $12: 59: 00.36$ & $29: 07: 50.3$ & 0.0618 & 32 & $\mathrm{c}$ & 5 & 18.56 & 0.92 & 3 & 0 & 23.57 \\
\hline 4194 & $12: 59: 00.65$ & $29: 28: 36.4$ & 0.1805 & 32 & $\mathrm{e}$ & 5 & 19.72 & 0.84 & 0 & 0 & 24.29 \\
\hline 4225 & $12: 59: 06.37$ & $28: 57: 12.9$ & 0.1486 & 90 & $\mathrm{x}$ & 5 & 19.95 & 1.40 & 0 & 0 & 23.82 \\
\hline 4237 & $12: 59: 08.03$ & $28: 56: 10.2$ & 0.1803 & 69 & $\mathrm{x}$ & 5 & 19.32 & 1.40 & 0 & 0 & 24.11 \\
\hline 4261 & $12: 59: 10.95$ & $29: 26: 52.4$ & 0.0239 & 51 & $\mathrm{e}$ & 4 & 20.09 & 0.47 & 0 & 0 & 24.64 \\
\hline 4270 & $12: 59: 12.10$ & $29: 01: 09.6$ & 0.0868 & 36 & $\mathrm{c}$ & 5 & 18.56 & 1.31 & 1 & 0 & 23.67 \\
\hline 4274 & $12: 59: 12.39$ & $29: 07: 13.1$ & 0.1636 & 26 & $\mathrm{e}$ & 4 & 19.79 & 0.88 & 0 & 0 & 24.17 \\
\hline 4312 & $12: 59: 17.67$ & $29: 02: 40.5$ & 0.2624 & 120 & $\mathrm{x}$ & 0 & 19.81 & 1.25 & 0 & 0 & 24.39 \\
\hline 4332 & $12: 59: 20.91$ & $29: 15: 36.1$ & 0.1794 & 52 & $\mathrm{x}$ & 4 & 19.82 & 1.39 & 0 & 0 & 24.10 \\
\hline 4374 & $12: 59: 26.15$ & $29: 08: 45.1$ & 0.0614 & 32 & $\mathrm{c}$ & 5 & 17.33 & 0.80 & 4 & 0 & 23.44 \\
\hline 4380 & $12: 59: 26.55$ & 29:08:09.6 & 0.1662 & 41 & $\mathrm{c}$ & 4 & 20.14 & 0.91 & 0 & 0 & 23.93 \\
\hline 4465 & $12: 59: 35.15$ & $29: 10: 16.3$ & 0.0602 & 106 & $\mathrm{x}$ & 1 & 19.13 & 1.06 & 0 & 0 & 24.82 \\
\hline 4470 & $12: 59: 36.02$ & $29: 16: 18.7$ & 0.0272 & 22 & $\mathrm{e}$ & 4 & 19.35 & 0.71 & 0 & 0 & 23.58 \\
\hline 4472 & $12: 59: 36.00$ & $29: 26: 37.2$ & 0.0944 & 91 & $\mathrm{x}$ & 5 & 19.75 & 1.26 & 0 & 0 & 24.28 \\
\hline 4488 & $12: 59: 37.56$ & $29: 27: 56.7$ & 0.0955 & 306 & $\mathrm{e}$ & 3 & 19.71 & 0.92 & 0 & 0 & 24.42 \\
\hline 4489 & $12: 59: 37.67$ & $29: 16: 39.2$ & 0.1783 & 99 & $\mathrm{x}$ & 4 & 19.25 & 1.04 & 0 & 0 & 24.74 \\
\hline 4492 & $12: 59: 38.25$ & $29: 02: 23.4$ & 0.0850 & 74 & $\mathrm{x}$ & 1 & 18.84 & 0.78 & 0 & 0 & 24.43 \\
\hline 4500 & $12: 59: 38.96$ & $29: 07: 16.4$ & 0.1621 & 79 & $\mathrm{x}$ & 5 & 19.70 & 1.42 & 0 & 0 & 23.75 \\
\hline 4503 & $12: 59: 39.02$ & $29: 06: 19.6$ & 0.1651 & 45 & $\mathrm{x}$ & 5 & 18.14 & 1.50 & 0 & 0 & 24.84 \\
\hline 4528 & $12: 59: 41.33$ & $29: 05: 55.0$ & 0.1670 & 41 & $\mathrm{x}$ & 5 & 19.12 & 1.42 & 0 & 0 & 23.96 \\
\hline 4577 & $12: 59: 46.04$ & $29: 13: 29.9$ & 0.1812 & 94 & $\mathrm{x}$ & 5 & 18.78 & 1.54 & 0 & 0 & 24.34 \\
\hline 4584 & $12: 59: 47.08$ & $29: 05: 58.7$ & 0.1661 & 70 & $\mathrm{x}$ & 4 & 19.63 & 1.48 & 0 & 0 & 24.29 \\
\hline 4585 & $12: 59: 47.03$ & $29: 25: 52.9$ & 0.1755 & 71 & $\mathrm{x}$ & 5 & 19.59 & 1.33 & 0 & 0 & 24.17 \\
\hline 4604 & $12: 59: 50.16$ & $28: 55: 54.5$ & 0.1793 & 29 & $\mathrm{e}$ & 3 & 19.53 & 0.97 & 0 & 0 & 24.05 \\
\hline 4614 & $12: 59: 51.08$ & $29: 08: 45.3$ & 0.0612 & 17 & $\mathrm{e}$ & 5 & 18.50 & 0.99 & 1 & 0 & 23.40 \\
\hline 4622 & $12: 59: 52.30$ & $29: 01: 53.2$ & 0.0830 & 5 & $\mathrm{e}$ & 4 & 19.23 & 0.49 & 0 & 0 & 23.91 \\
\hline 4631 & $12: 59: 53.52$ & $29: 06: 16.6$ & 0.1686 & 46 & $\mathrm{x}$ & 5 & 19.82 & 0.98 & 0 & 0 & 24.09 \\
\hline 4632 & $12: 59: 53.53$ & $29: 13: 58.6$ & 0.0238 & 40 & $\mathrm{e}$ & 2 & 19.38 & 0.28 & 0 & 0 & 24.66 \\
\hline 4653 & $12: 59: 55.75$ & $28: 57: 06.0$ & 0.0840 & 4 & $\mathrm{e}$ & 5 & 18.84 & 0.86 & 0 & 0 & 23.78 \\
\hline 4669 & $12: 59: 57.92$ & $28: 59: 56.0$ & 0.1632 & 47 & $\mathrm{x}$ & 5 & 19.56 & 1.42 & 0 & 0 & 24.04 \\
\hline 4676 & $12: 59: 59.05$ & $29: 03: 48.9$ & 0.1681 & 11 & $\mathrm{e}$ & 2 & 19.71 & 0.88 & 0 & 0 & 23.99 \\
\hline 4687 & 13:00:00.66 & $29: 10: 16.0$ & 0.0614 & 22 & $\mathrm{e}$ & 4 & 18.82 & 0.66 & 0 & 0 & 23.73 \\
\hline 4707 & 13:00:02.39 & $29: 31: 45.3$ & 0.1661 & 60 & $\mathrm{x}$ & 5 & 19.34 & 1.20 & 0 & 0 & 24.23 \\
\hline 4718 & $13: 00: 04.16$ & $29: 23: 05.8$ & 0.2274 & 56 & $\mathrm{x}$ & 5 & 19.65 & 1.38 & 0 & 0 & 24.17 \\
\hline 4741 & 13:00:06.78 & $29: 01: 43.3$ & 0.1712 & 21 & $\mathrm{e}$ & 5 & 19.48 & 0.97 & 0 & 0 & 23.75 \\
\hline 4755 & 13:00:08.21 & 29:09:49.9 & 0.0617 & 5 & $\mathrm{e}$ & 5 & 19.42 & 0.78 & 0 & 0 & 23.63 \\
\hline 4788 & $13: 00: 12.44$ & $29: 11: 46.1$ & 0.1811 & 70 & $\mathrm{x}$ & 3 & 19.99 & 1.35 & 0 & 0 & 24.13 \\
\hline 4801 & $13: 00: 14.00$ & $28: 49: 40.8$ & 0.0246 & 37 & $\mathrm{x}$ & 5 & 15.95 & 1.04 & 3 & 1 & 24.03 \\
\hline 4826 & $13: 00: 18.16$ & $29: 10: 32.8$ & 0.1687 & 98 & $\mathrm{x}$ & 3 & 19.63 & 1.06 & 0 & 0 & 24.06 \\
\hline 4862 & $13: 00: 21.30$ & $29: 34: 48.1$ & 0.0851 & 31 & $\mathrm{c}$ & 5 & 18.42 & 1.33 & 0 & 0 & 24.15 \\
\hline 4865 & $13: 00: 22.42$ & 29:09:16.7 & 0.1799 & 38 & $\mathrm{e}$ & 3 & 19.34 & 0.97 & 0 & 0 & 23.99 \\
\hline 4891 & $13: 00: 25.05$ & $29: 31: 46.2$ & 0.1943 & 35 & $\mathrm{e}$ & 4 & 19.65 & 1.07 & 0 & 0 & 24.14 \\
\hline 4917 & $13: 00: 28.44$ & $29: 22: 25.6$ & 0.0855 & 75 & $\mathrm{x}$ & 3 & 19.72 & 1.27 & 0 & 0 & 23.97 \\
\hline 4922 & $13: 00: 29.16$ & 29:09:12.1 & 0.0859 & 21 & $\mathrm{e}$ & 4 & 18.60 & 0.53 & 0 & 0 & 24.41 \\
\hline 4936 & $13: 00: 30.74$ & $29: 20: 28.1$ & 0.0882 & 38 & $\mathrm{c}$ & 5 & 18.53 & 0.82 & 0 & 0 & 23.62 \\
\hline 4938 & 13:00:31.19 & $28: 57: 02.0$ & 0.0230 & 18 & $\mathrm{e}$ & 4 & 18.41 & 0.69 & 0 & 0 & 24.62 \\
\hline 4975 & $13: 00: 39.67$ & $29: 01: 10.0$ & 0.0244 & 28 & $\mathrm{x}$ & 5 & 14.96 & 0.80 & 6 & 1 & 23.43 \\
\hline 4988 & $13: 00: 41.73$ & $29: 17: 13.4$ & 0.1802 & 29 & $\mathrm{e}$ & 5 & 18.90 & 1.31 & 0 & 0 & 23.81 \\
\hline 5014 & $13: 00: 45.97$ & $29: 17: 18.6$ & 0.1783 & 63 & $\mathrm{x}$ & 4 & 19.68 & 1.48 & 0 & 0 & 24.15 \\
\hline 5024 & $13: 00: 47.57$ & $29: 12: 02.3$ & 0.0310 & 29 & $\mathrm{x}$ & 5 & 17.99 & 1.12 & 2 & 0 & 23.62 \\
\hline 5075 & $13: 00: 54.82$ & $29: 17: 50.3$ & 0.1811 & 32 & $\mathrm{e}$ & 4 & 19.81 & 1.04 & 0 & 0 & 24.11 \\
\hline 5121 & 13:01:00.19 & $29: 06: 10.0$ & 0.1925 & 72 & $\mathrm{x}$ & 4 & 20.05 & 1.51 & 0 & 0 & 24.71 \\
\hline 5143 & $13: 01: 02.47$ & $28: 58: 20.8$ & 0.0899 & 31 & $\mathrm{c}$ & 3 & 19.47 & 1.22 & 0 & 0 & 24.26 \\
\hline 5149 & $13: 01: 02.80$ & $29: 23: 41.8$ & 0.1918 & 30 & $\mathrm{c}$ & 5 & 18.99 & 0.95 & 0 & 0 & 23.90 \\
\hline 5153 & $13: 01: 03.25$ & $29: 03: 15.6$ & 0.2577 & 103 & $\mathrm{x}$ & 1 & 20.71 & 1.40 & 0 & 0 & 24.39 \\
\hline 5167 & $13: 01: 04.71$ & $29: 16: 17.4$ & 0.1801 & 57 & $\mathrm{c}$ & 3 & 19.31 & 1.05 & 0 & 0 & 24.51 \\
\hline 5183 & 13:01:07.01 & $28: 56: 06.0$ & 0.1648 & 41 & $\mathrm{x}$ & 3 & 19.48 & 1.41 & 0 & 0 & 24.29 \\
\hline 5207 & $13: 01: 09.25$ & $29: 28: 44.2$ & 0.0839 & 30 & $\mathrm{c}$ & 5 & 18.06 & 0.71 & 0 & 0 & 24.32 \\
\hline 5208 & 13:01:09.49 & $29: 03: 54.8$ & 0.0279 & 19 & $\mathrm{e}$ & 5 & 18.31 & 0.26 & 0 & 0 & 24.25 \\
\hline 5217 & $13: 01: 10.58$ & $29: 21: 11.9$ & 0.0881 & 65 & $\mathrm{c}$ & 5 & 20.01 & 1.44 & 0 & 0 & 24.81 \\
\hline 5218 & $13: 01: 10.80$ & $28: 56: 27.1$ & 0.1933 & 5 & $\mathrm{e}$ & 4 & 19.91 & 0.92 & 0 & 0 & 24.31 \\
\hline 5237 & $13: 01: 12.74$ & $29: 17: 50.6$ & 0.1780 & 61 & $\mathrm{x}$ & 5 & 19.42 & 1.21 & 0 & 0 & 24.31 \\
\hline 5250 & $13: 01: 13.75$ & $29: 04: 25.1$ & 0.1651 & 67 & $\mathrm{x}$ & 4 & 19.36 & 1.47 & 0 & 0 & 24.17 \\
\hline 5254 & $13: 01: 13.98$ & $29: 07: 52.0$ & 0.1919 & 77 & $\mathrm{x}$ & 2 & 20.00 & 1.47 & 0 & 0 & 23.88 \\
\hline 5260 & $13: 01: 14.75$ & $29: 08: 34.3$ & 0.0618 & 39 & $\mathrm{e}$ & 2 & 18.84 & 0.89 & 0 & 0 & 23.95 \\
\hline
\end{tabular}


TABLE 3-Continued

\begin{tabular}{|c|c|c|c|c|c|c|c|c|c|c|c|}
\hline $\begin{array}{r}\text { NSER } \\
(1)\end{array}$ & $\begin{array}{l}\text { R.A. } \\
2000.0 \\
\quad(2)\end{array}$ & $\begin{array}{l}\text { Dec. } \\
2000.0 \\
(3)\end{array}$ & (4) & $\begin{array}{c} \pm \\
\mathrm{kms}^{-1} \\
(5)\end{array}$ & (6) & $\begin{array}{l}\mathrm{q} \\
(7)\end{array}$ & $\begin{array}{l}\mathrm{b}_{J} \\
(8)\end{array}$ & $\begin{array}{c}\mathrm{b}_{J}-\mathrm{r}_{F} \\
(9)\end{array}$ & $\begin{array}{c}\operatorname{sat}\left(\mathrm{b}_{J}\right) \\
\% \\
(10)\end{array}$ & $\begin{array}{c}\operatorname{sat}\left(\mathrm{r}_{F}\right) \\
\% \\
(11)\end{array}$ & $\begin{array}{c}\mathrm{SB} \\
\mathrm{b}_{J} \operatorname{arcsec}^{-2} \\
(12)\end{array}$ \\
\hline 5262 & 13:01:14.99 & $29: 02: 34.5$ & 0.1647 & 11 & $\mathrm{e}$ & 3 & 19.57 & 1.09 & 0 & 0 & 24.09 \\
\hline 5269 & $13: 01: 15.37$ & $29: 22: 11.8$ & 0.0882 & 45 & $\mathrm{x}$ & 5 & 18.05 & 1.22 & 1 & 0 & 24.26 \\
\hline 5272 & $13: 01: 15.72$ & $28: 52: 19.3$ & 0.0238 & 36 & $\mathrm{x}$ & 4 & 18.08 & 1.18 & 5 & 0 & 23.89 \\
\hline 5273 & $13: 01: 15.47$ & $29: 32: 10.5$ & 0.1912 & 67 & $\mathrm{x}$ & 4 & 20.03 & 1.44 & 0 & 0 & 23.98 \\
\hline 5274 & $13: 01: 15.74$ & 29:01:06.0 & 0.1663 & 65 & $\mathrm{x}$ & 3 & 18.52 & 1.17 & 0 & 0 & 24.14 \\
\hline 5277 & $13: 01: 15.80$ & $29: 17: 39.6$ & 0.1802 & 75 & $\mathrm{x}$ & 5 & 19.96 & 1.33 & 0 & 0 & 24.21 \\
\hline 5283 & $13: 01: 16.26$ & $29: 16: 55.3$ & 0.1797 & 101 & $\mathrm{x}$ & 3 & 19.87 & 1.02 & 0 & 0 & 24.49 \\
\hline 5284 & $13: 01: 16.34$ & $29: 17: 52.7$ & 0.1797 & 64 & $\mathrm{x}$ & 4 & 19.65 & 1.41 & 0 & 0 & 24.18 \\
\hline 5289 & $13: 01: 17.12$ & $29: 02: 33.6$ & 0.1647 & 4 & $\mathrm{e}$ & 5 & 19.27 & 1.11 & 0 & 0 & 23.75 \\
\hline 5324 & $13: 01: 19.51$ & $29: 10: 59.5$ & 0.0611 & 21 & $\mathrm{e}$ & 3 & 17.59 & 0.69 & 0 & 0 & 24.18 \\
\hline 5356 & $13: 01: 21.97$ & $29: 20: 22.9$ & 0.0877 & 41 & $\mathrm{x}$ & 6 & 18.39 & 1.30 & 1 & 0 & 24.09 \\
\hline 5364 & $13: 01: 22.95$ & $28: 49: 54.8$ & 0.1343 & 12 & $\mathrm{e}$ & 3 & 19.50 & 0.88 & 0 & 0 & 24.18 \\
\hline 5367 & $13: 01: 23.12$ & 29:00:35.0 & 0.0896 & 60 & $\mathrm{x}$ & 5 & 18.38 & 1.35 & 1 & 0 & 23.96 \\
\hline 5374 & $13: 01: 23.44$ & $29: 04: 18.2$ & 0.1918 & 75 & $x$ & 3 & 19.31 & 1.49 & 0 & 0 & 24.66 \\
\hline 5381 & $13: 01: 24.26$ & $29: 22: 51.9$ & 0.1789 & 10 & $\mathrm{e}$ & 3 & 19.34 & 1.09 & 0 & 0 & 24.14 \\
\hline 5387 & $13: 01: 24.88$ & $29: 29: 19.3$ & 0.1925 & 123 & $\mathrm{x}$ & 4 & 20.15 & 1.44 & 0 & 0 & 24.05 \\
\hline 5399 & $13: 01: 26.53$ & $29: 07: 34.0$ & 0.1927 & 72 & $\mathrm{x}$ & 4 & 19.34 & 1.12 & 1 & 0 & 24.32 \\
\hline 5402 & $13: 01: 26.88$ & 29:04:00.8 & 0.1651 & 69 & $\mathrm{x}$ & 5 & 20.28 & 1.49 & 0 & 0 & 23.85 \\
\hline 5405 & $13: 01: 27.45$ & $28: 54: 55.9$ & 0.1923 & 73 & $\mathrm{x}$ & 3 & 20.12 & 1.51 & 0 & 0 & 24.50 \\
\hline 5410 & $13: 01: 27.85$ & $29: 22: 05.9$ & 0.0874 & 34 & $\mathrm{c}$ & 5 & 19.21 & 0.97 & 0 & 0 & 23.68 \\
\hline 5415 & $13: 01: 28.45$ & 29:03:50.1 & 0.0825 & 5 & $\mathrm{e}$ & 4 & 19.36 & 0.77 & 0 & 0 & 23.69 \\
\hline 5419 & $13: 01: 28.69$ & $29: 20: 52.5$ & 0.0883 & 57 & $\mathrm{x}$ & 2 & 19.53 & 0.98 & 0 & 0 & 23.99 \\
\hline 5423 & $13: 01: 29.09$ & $28: 59: 33.4$ & 0.1644 & 32 & $\mathrm{c}$ & 5 & 19.46 & 1.04 & 0 & 0 & 23.65 \\
\hline 5424 & $13: 01: 29.14$ & $29: 05: 24.0$ & 0.1924 & 53 & $\mathrm{x}$ & 4 & 19.62 & 1.40 & 0 & 0 & 24.09 \\
\hline 5429 & $13: 01: 29.50$ & $29: 23: 11.3$ & 0.1799 & 23 & $\mathrm{e}$ & 1 & 19.37 & 0.90 & 0 & 0 & 24.31 \\
\hline 5465 & $13: 01: 33.72$ & $29: 07: 50.1$ & 0.0250 & 29 & $\mathrm{x}$ & 5 & 15.29 & 1.07 & 5 & 3 & 23.86 \\
\hline 5470 & $13: 01: 34.06$ & $28: 58: 55.4$ & 0.0825 & 28 & $\mathrm{c}$ & 5 & 18.69 & 1.12 & 1 & 0 & 23.64 \\
\hline 5483 & $13: 01: 34.96$ & $29: 09: 31.9$ & 0.1909 & 45 & $\mathrm{x}$ & 5 & 19.67 & 1.48 & 0 & 0 & 24.40 \\
\hline 5486 & $13: 01: 35.36$ & $29: 05: 11.2$ & 0.1673 & 85 & $\mathrm{x}$ & 4 & 19.54 & 1.42 & 0 & 0 & 24.09 \\
\hline 5487 & $13: 01: 35.32$ & $29: 11: 53.8$ & 0.1907 & 26 & $\mathrm{e}$ & 2 & 19.87 & 1.31 & 0 & 0 & 24.11 \\
\hline 5497 & $13: 01: 36.29$ & $29: 13: 42.0$ & 0.1802 & 18 & $\mathrm{e}$ & 5 & 19.84 & 0.90 & 0 & 0 & 23.78 \\
\hline 5509 & $13: 01: 38.03$ & $28: 59: 13.1$ & 0.0825 & 52 & $\mathrm{x}$ & 5 & 19.04 & 1.19 & 0 & 0 & 24.11 \\
\hline 5514 & $13: 01: 38.60$ & $28: 49: 10.8$ & 0.1849 & 29 & $\mathrm{e}$ & 3 & 19.81 & 1.46 & 0 & 0 & 24.14 \\
\hline 5539 & $13: 01: 41.51$ & $29: 22: 52.1$ & 0.0574 & 16 & $\mathrm{e}$ & 5 & 17.83 & 0.82 & 3 & 0 & 23.62 \\
\hline 5549 & $13: 01: 42.38$ & $29: 04: 49.6$ & 0.1634 & 59 & $\mathrm{x}$ & 4 & 19.10 & 1.41 & 0 & 0 & 24.97 \\
\hline 5561 & $13: 01: 43.33$ & $29: 02: 39.9$ & 0.0235 & 28 & $\mathrm{c}$ & 5 & 15.29 & 0.87 & 6 & 2 & 23.75 \\
\hline 5562 & $13: 01: 43.28$ & $29: 10: 41.3$ & 0.0236 & 31 & $\mathrm{x}$ & 5 & 18.01 & 0.97 & 2 & 0 & 23.99 \\
\hline 5573 & $13: 01: 43.95$ & $28: 59: 57.7$ & 0.0213 & 31 & $x$ & 4 & 16.84 & 1.12 & 4 & 1 & 23.88 \\
\hline 5592 & $13: 01: 46.41$ & 29:04:33.0 & 0.0615 & 22 & $\mathrm{e}$ & 3 & 17.94 & 0.42 & 0 & 0 & 24.70 \\
\hline 5597 & $13: 01: 46.69$ & $29: 05: 12.6$ & 0.1670 & 52 & $\mathrm{x}$ & 4 & 19.34 & 1.37 & 0 & 0 & 24.15 \\
\hline 5598 & $13: 01: 46.53$ & $29: 29: 08.6$ & 0.1919 & 78 & $\mathrm{x}$ & 5 & 20.00 & 1.54 & 0 & 0 & 24.23 \\
\hline 5618 & $13: 01: 48.93$ & $28: 58: 60.0$ & 0.2866 & 20 & $\mathrm{e}$ & 5 & 19.91 & 0.77 & 0 & 0 & 23.74 \\
\hline 5646 & $13: 01: 51.75$ & $29: 08: 14.9$ & 0.1650 & 90 & $\mathrm{x}$ & 4 & 19.56 & 1.32 & 0 & 0 & 23.87 \\
\hline 5677 & $13: 01: 55.79$ & $29: 19: 21.2$ & 0.0236 & 26 & $\mathrm{x}$ & 5 & 17.36 & 1.12 & 4 & 1 & 23.71 \\
\hline 5679 & $13: 01: 56.29$ & $28: 49: 11.4$ & 0.1345 & 31 & $\mathrm{e}$ & 2 & 19.56 & 1.17 & 0 & 0 & 24.00 \\
\hline 5702 & $13: 01: 58.51$ & $29: 06: 17.3$ & 0.1645 & 99 & $\mathrm{x}$ & 4 & 19.22 & 1.48 & 0 & 0 & 24.11 \\
\hline 5703 & $13: 01: 58.57$ & $29: 11: 13.5$ & 0.0831 & 5 & $\mathrm{e}$ & 4 & 18.99 & 0.70 & 0 & 0 & 23.46 \\
\hline 5717 & $13: 02: 00.22$ & $29: 04: 47.1$ & 0.1634 & 70 & $\mathrm{x}$ & 5 & 19.80 & 1.43 & 0 & 0 & 23.94 \\
\hline 5719 & $13: 02: 00.42$ & $28: 58: 46.0$ & 1.7700 & 0 & $\mathrm{e}$ & 3 & 19.40 & 0.65 & 0 & 0 & 23.62 \\
\hline 5726 & $13: 02: 00.86$ & $28: 57: 24.8$ & 0.1925 & 18 & e & 4 & 18.87 & 0.86 & 0 & 0 & 23.71 \\
\hline 5727 & 13:02:00.96 & $29: 30: 36.2$ & 0.0480 & 31 & $\mathrm{c}$ & 5 & 18.48 & 1.10 & 0 & 0 & 23.64 \\
\hline 5745 & $13: 02: 03.14$ & $29: 05: 55.1$ & 0.1651 & 83 & $\mathrm{x}$ & 4 & 20.39 & 1.38 & 0 & 0 & 23.99 \\
\hline 5763 & $13: 02: 04.16$ & $29: 15: 12.0$ & 0.0244 & 21 & $\mathrm{x}$ & 5 & 16.31 & 1.21 & 8 & 4 & 23.63 \\
\hline 5764 & $13: 02: 04.37$ & $28: 53: 39.8$ & 0.0269 & 23 & $\mathrm{x}$ & 5 & 16.80 & 1.17 & 4 & 2 & 23.58 \\
\hline 5776 & $13: 02: 05.53$ & $29: 06: 44.2$ & 0.0492 & 6 & $\mathrm{e}$ & 5 & 18.16 & 0.81 & 1 & 0 & 23.37 \\
\hline 5817 & $13: 02: 10.16$ & $29: 24: 46.9$ & 0.1710 & 41 & $\mathrm{x}$ & 4 & 19.45 & 1.00 & 0 & 0 & 24.19 \\
\hline 5843 & $13: 02: 13.61$ & $29: 14: 05.6$ & 0.1639 & 64 & $\mathrm{x}$ & 5 & 19.52 & 1.36 & 0 & 0 & 23.82 \\
\hline 5862 & $13: 02: 16.12$ & $29: 23: 12.4$ & 0.1936 & 22 & $\mathrm{e}$ & 3 & 20.00 & 0.82 & 0 & 0 & 24.06 \\
\hline 5871 & $13: 02: 17.00$ & $29: 16: 43.8$ & 0.0870 & 28 & $\mathrm{c}$ & 5 & 18.07 & $\ldots$ & 19 & $\ldots$ & 23.48 \\
\hline 5899 & $13: 02: 19.38$ & $28: 57: 09.0$ & 0.0346 & 28 & $\mathrm{x}$ & 5 & 17.60 & 1.20 & 3 & 1 & 23.48 \\
\hline 5905 & $13: 02: 20.03$ & $29: 12: 12.8$ & 0.1664 & 88 & $\mathrm{x}$ & 4 & 19.34 & 1.23 & 0 & 0 & 23.96 \\
\hline 5923 & $13: 02: 21.87$ & $28: 51: 44.9$ & 0.0836 & 6 & $\mathrm{e}$ & 3 & 18.85 & 0.46 & 0 & 0 & 24.39 \\
\hline 5931 & $13: 02: 22.78$ & $29: 08: 57.6$ & 0.0495 & 33 & $\mathrm{c}$ & 3 & 18.51 & 0.56 & 0 & 0 & 24.03 \\
\hline 5947 & $13: 02: 24.32$ & $29: 27: 05.4$ & 0.0265 & 82 & $\mathrm{x}$ & 2 & 18.64 & 1.01 & 1 & 0 & 23.97 \\
\hline 5984 & $13: 02: 27.74$ & $29: 29: 14.8$ & 0.0237 & 27 & $\mathrm{x}$ & 5 & 18.17 & 1.08 & 1 & 0 & 23.75 \\
\hline 5997 & $13: 02: 29.47$ & $29: 12: 34.1$ & 0.0892 & 26 & $\mathrm{e}$ & 5 & 17.78 & 0.84 & 0 & 0 & 24.08 \\
\hline 6053 & $13: 02: 37.68$ & $29: 06: 29.8$ & 0.0839 & 75 & $\mathrm{x}$ & 4 & 18.97 & 1.10 & 0 & 0 & 23.68 \\
\hline 6074 & $13: 02: 40.55$ & $28: 53: 49.0$ & 0.1909 & 4 & $\mathrm{e}$ & 5 & 19.63 & 0.89 & 0 & 0 & 23.76 \\
\hline 6080 & $13: 02: 40.94$ & 29:00:60.0 & 0.0347 & 20 & $\mathrm{e}$ & 3 & 19.14 & 0.48 & 0 & 0 & 24.14 \\
\hline 6089 & $13: 02: 42.44$ & $29: 03: 22.9$ & 0.0839 & 32 & $\mathrm{e}$ & 3 & 19.77 & 0.58 & 0 & 0 & 24.28 \\
\hline
\end{tabular}


TABLE 3-Continued

\begin{tabular}{|c|c|c|c|c|c|c|c|c|c|c|c|}
\hline $\begin{array}{r}\text { NSER } \\
(1)\end{array}$ & $\begin{array}{l}\text { R.A. } \\
2000.0 \\
\quad(2)\end{array}$ & $\begin{array}{l}\text { Dec. } \\
2000.0 \\
(3)\end{array}$ & (4) & $\begin{array}{c} \pm \\
\mathrm{kms}^{-1} \\
(5)\end{array}$ & (6) & (7) & $\begin{array}{l}\mathrm{b}_{J} \\
(8)\end{array}$ & $\begin{array}{c}\mathrm{b}_{J}-\mathrm{r}_{F} \\
(9)\end{array}$ & $\begin{array}{c}\operatorname{sat}\left(\mathrm{b}_{J}\right) \\
\% \\
(10)\end{array}$ & $\begin{array}{c}\operatorname{sat}\left(\mathrm{r}_{F}\right) \\
\% \\
(11)\end{array}$ & $\begin{array}{c}\mathrm{SB} \\
\mathrm{b}_{J} \operatorname{arcsec}^{-2} \\
(12)\end{array}$ \\
\hline 6093 & $13: 02: 42.55$ & $29: 07: 46.0$ & 0.1643 & 50 & $\mathrm{x}$ & 5 & 19.24 & 1.35 & 0 & 0 & 23.94 \\
\hline 6104 & $13: 02: 43.46$ & $29: 26: 05.7$ & 0.0892 & 31 & $\mathrm{c}$ & 5 & 18.26 & 0.91 & 1 & 0 & 23.59 \\
\hline 6106 & $13: 02: 43.61$ & $29: 30: 55.2$ & 0.1667 & 75 & $\mathrm{x}$ & 5 & 20.30 & 1.35 & 0 & 0 & 24.71 \\
\hline 6117 & $13: 02: 45.19$ & $29: 05: 22.2$ & 0.0831 & 14 & $\mathrm{e}$ & 3 & 19.65 & 0.70 & 0 & 0 & 24.15 \\
\hline 6125 & $13: 02: 46.25$ & $28: 59: 44.7$ & 0.1977 & 67 & $\mathrm{x}$ & 5 & 19.62 & 1.23 & 0 & 0 & 23.90 \\
\hline 6148 & $13: 02: 48.21$ & $29: 07: 48.8$ & 0.0831 & 33 & $\mathrm{c}$ & 4 & 18.57 & 1.26 & 1 & 0 & 23.69 \\
\hline 6152 & $13: 02: 49.06$ & $29: 05: 22.7$ & 0.0831 & 33 & $\mathrm{c}$ & 5 & 17.84 & 0.99 & 1 & 0 & 23.59 \\
\hline 6182 & $13: 02: 52.25$ & $29: 07: 45.6$ & 0.1578 & 33 & $\mathrm{e}$ & 2 & 20.61 & 0.62 & 0 & 0 & 24.22 \\
\hline 6196 & $13: 02: 54.19$ & 29:06:01.1 & 0.1667 & 52 & $\mathrm{x}$ & 4 & 19.18 & 1.04 & 0 & 0 & 24.17 \\
\hline 6303 & $13: 03: 09.77$ & $28: 54: 51.5$ & 0.3255 & 52 & $\mathrm{x}$ & 4 & 20.62 & 1.81 & 0 & 0 & 24.49 \\
\hline 6315 & $13: 03: 11.34$ & $29: 27: 57.6$ & 0.0841 & 53 & $\mathrm{x}$ & 4 & 19.07 & 1.02 & 0 & 0 & 24.03 \\
\hline 6322 & $13: 03: 12.08$ & $28: 54: 19.3$ & 0.1789 & 66 & $\mathrm{x}$ & 3 & 20.14 & 1.35 & 0 & 0 & 24.12 \\
\hline 6329 & $13: 03: 12.68$ & $29: 18: 09.5$ & 0.0838 & 33 & $\mathrm{c}$ & 5 & 18.54 & 1.06 & 1 & 0 & 23.53 \\
\hline 6355 & $13: 03: 15.80$ & $29: 13: 44.4$ & 0.2171 & 19 & $\mathrm{e}$ & 4 & 19.67 & 0.80 & 0 & 0 & 23.90 \\
\hline 6363 & $13: 03: 17.22$ & $29: 15: 36.0$ & 0.0817 & 20 & $\mathrm{e}$ & 3 & 18.71 & 0.80 & 0 & 0 & 23.65 \\
\hline 6457 & $13: 03: 28.94$ & $28: 59: 17.1$ & 0.0836 & 32 & $\mathrm{x}$ & 5 & 17.78 & 1.09 & 1 & 0 & 23.70 \\
\hline 6484 & $13: 03: 31.94$ & $28: 58: 46.6$ & 0.1834 & 29 & $\mathrm{e}$ & 3 & 20.56 & 1.15 & 0 & 0 & 24.69 \\
\hline 6487 & $13: 03: 32.14$ & $29: 06: 46.8$ & 0.1836 & 104 & $\mathrm{e}$ & 5 & 19.77 & 1.08 & 0 & 0 & 24.05 \\
\hline 6512 & $13: 03: 35.60$ & $29: 31: 45.7$ & 0.2247 & 91 & $\mathrm{x}$ & 5 & 19.77 & 1.49 & 0 & 0 & 24.22 \\
\hline 6530 & $13: 03: 37.24$ & $29: 17: 58.6$ & 0.0892 & 70 & $\mathrm{x}$ & 5 & 18.80 & 0.97 & 0 & 0 & 23.79 \\
\hline 6564 & $13: 03: 40.96$ & $28: 56: 24.8$ & 0.2369 & 110 & $\mathrm{x}$ & 4 & 20.48 & 1.62 & 0 & 0 & 24.79 \\
\hline 6571 & $13: 03: 42.18$ & $28: 54: 18.9$ & 0.0223 & 37 & $\mathrm{x}$ & 5 & 17.21 & 1.10 & 3 & 1 & 23.92 \\
\hline 6607 & $13: 03: 47.59$ & $29: 13: 13.9$ & 0.1668 & 94 & $\mathrm{x}$ & 5 & 19.16 & 1.26 & 0 & 0 & 23.91 \\
\hline 6622 & $13: 03: 49.12$ & 29:08:30.1 & 0.0618 & 31 & $\mathrm{c}$ & 5 & 18.63 & 1.17 & 0 & 0 & 23.89 \\
\hline 6643 & $13: 03: 51.57$ & $29: 21: 21.7$ & 0.2279 & 60 & $\mathrm{x}$ & 5 & 19.84 & 1.47 & 0 & 0 & 24.18 \\
\hline 6645 & $13: 03: 52.05$ & $29: 11: 17.9$ & 0.0825 & 41 & $\mathrm{x}$ & 5 & 18.51 & 1.31 & 1 & 0 & 23.76 \\
\hline 6679 & $13: 03: 56.60$ & $29: 23: 04.0$ & 0.2130 & 45 & $\mathrm{e}$ & 5 & 20.04 & 1.31 & 0 & 0 & 24.29 \\
\hline 6712 & $13: 04: 00.59$ & $29: 04: 25.3$ & 0.0815 & 27 & $\mathrm{c}$ & 5 & 18.92 & 1.01 & 0 & 0 & 23.68 \\
\hline 6754 & $13: 04: 04.39$ & $29: 02: 06.5$ & 0.0622 & 22 & $\mathrm{e}$ & 5 & 19.47 & 0.79 & 0 & 0 & 23.82 \\
\hline 6793 & $13: 04: 09.57$ & $29: 04: 24.3$ & 0.1917 & 60 & $\mathrm{x}$ & 5 & 19.21 & 1.23 & 0 & 0 & 24.18 \\
\hline 6800 & $13: 04: 10.04$ & $29: 16: 54.7$ & 0.1395 & 24 & $\mathrm{e}$ & 4 & 19.52 & 0.65 & 0 & 0 & 24.14 \\
\hline 6802 & $13: 04: 10.18$ & 29:00:55.0 & 0.0220 & 19 & $\mathrm{x}$ & 5 & 17.22 & 1.02 & 1 & 0 & 24.25 \\
\hline 6854 & $13: 04: 17.88$ & $29: 01: 45.8$ & 0.0228 & 19 & $\mathrm{x}$ & 5 & 16.29 & 1.02 & 5 & 1 & 23.75 \\
\hline 6886 & $13: 04: 23.26$ & $29: 08: 25.0$ & 0.1868 & 21 & $\mathrm{e}$ & 5 & 19.34 & 0.89 & 0 & 0 & 24.03 \\
\hline 6890 & $13: 04: 23.33$ & $29: 23: 00.0$ & 0.0893 & 32 & $\mathrm{x}$ & 5 & 18.03 & 1.25 & 2 & 0 & 23.95 \\
\hline 6891 & $13: 04: 23.37$ & $29: 25: 37.2$ & 0.1966 & 86 & $\mathrm{x}$ & 5 & 19.88 & 1.27 & 0 & 0 & 24.31 \\
\hline 6929 & $13: 04: 29.28$ & $28: 59: 14.3$ & 0.1088 & 65 & $\mathrm{x}$ & 2 & 19.13 & 0.82 & 0 & 0 & 24.33 \\
\hline 6932 & $13: 04: 29.61$ & $28: 55: 47.3$ & 0.0825 & 20 & $\mathrm{e}$ & 5 & 18.32 & 0.83 & 0 & 0 & 23.58 \\
\hline 6949 & $13: 04: 31.55$ & $29: 07: 04.7$ & 0.2150 & 84 & $\mathrm{x}$ & 5 & 20.57 & 1.59 & 0 & 0 & 24.09 \\
\hline 6984 & $13: 04: 37.02$ & $29: 08: 48.0$ & 0.0193 & 26 & $\mathrm{e}$ & 4 & 18.42 & 0.72 & 0 & 0 & 24.02 \\
\hline 6993 & $13: 04: 38.73$ & $28: 58: 21.3$ & 0.0253 & 29 & $\mathrm{e}$ & 4 & 17.73 & 0.83 & 1 & 0 & 23.65 \\
\hline 6996 & $13: 04: 38.95$ & $28: 59: 50.1$ & 0.1631 & 72 & $\mathrm{c}$ & 5 & 19.31 & 1.20 & 0 & 0 & 23.98 \\
\hline 6997 & $13: 04: 38.89$ & $29: 13: 28.6$ & 0.0192 & 26 & $\mathrm{c}$ & 5 & 17.75 & 1.07 & 0 & 0 & 24.14 \\
\hline 7078 & $13: 04: 50.31$ & $29: 13: 54.2$ & 0.1388 & 25 & $\mathrm{e}$ & 3 & 18.68 & 0.95 & 0 & 0 & 24.11 \\
\hline 7100 & $13: 04: 54.39$ & $29: 25: 37.4$ & 0.1865 & 86 & $\mathrm{x}$ & 4 & 17.71 & 1.44 & 7 & 1 & 23.64 \\
\hline 7130 & $13: 04: 58.32$ & $29: 11: 39.8$ & 0.0193 & 39 & $\mathrm{c}$ & 3 & 18.86 & 0.86 & 0 & 0 & 24.16 \\
\hline 7150 & $13: 05: 00.56$ & $29: 13: 06.9$ & 0.1976 & 81 & $\mathrm{x}$ & 4 & 20.43 & 1.43 & 0 & 0 & 24.79 \\
\hline 7155 & $13: 05: 01.12$ & $29: 16: 50.7$ & 0.1964 & 81 & $\mathrm{x}$ & 5 & 20.12 & 1.38 & 0 & 0 & 24.15 \\
\hline 7226 & $13: 05: 10.18$ & $29: 11: 13.9$ & 0.1963 & 73 & $\mathrm{x}$ & 5 & 19.92 & 1.40 & 0 & 0 & 24.00 \\
\hline 7250 & $13: 05: 12.29$ & $29: 14: 09.1$ & 0.0232 & 20 & $\mathrm{e}$ & 5 & 18.91 & 0.50 & 0 & 0 & 23.72 \\
\hline 7270 & $13: 05: 14.51$ & $29: 27: 46.2$ & 0.2399 & 36 & e & 2 & 19.87 & 0.95 & 0 & 0 & 24.23 \\
\hline 7280 & $13: 05: 15.59$ & 29:08:07.1 & 0.1869 & 34 & $\mathrm{c}$ & 5 & 18.86 & 1.10 & 0 & 0 & 23.93 \\
\hline 7302 & $13: 05: 17.74$ & $29: 07: 38.2$ & 0.0816 & 13 & $\mathrm{e}$ & 2 & 19.24 & 0.82 & 0 & 0 & 24.19 \\
\hline 7309 & $13: 05: 18.55$ & $29: 08: 30.6$ & 0.0818 & 44 & $\mathrm{x}$ & 3 & 18.46 & 1.23 & 0 & 0 & 23.95 \\
\hline 7318 & $13: 05: 19.53$ & $29: 11: 50.1$ & 0.0269 & 30 & $\mathrm{x}$ & 5 & 18.40 & 1.08 & 0 & 0 & 23.91 \\
\hline 7344 & $13: 05: 23.00$ & $29: 24: 52.6$ & 0.1703 & 94 & $\mathrm{x}$ & 5 & 19.61 & 1.33 & 0 & 0 & 24.16 \\
\hline 7366 & $13: 05: 25.21$ & $29: 17: 47.0$ & 0.0236 & 24 & $\mathrm{x}$ & 5 & 16.29 & 1.09 & 6 & 1 & 23.73 \\
\hline 7427 & $13: 05: 32.79$ & $29: 00: 40.7$ & 0.0175 & 30 & $\mathrm{c}$ & 4 & 17.06 & 0.94 & 3 & 0 & 24.09 \\
\hline 7449 & $13: 05: 34.96$ & $29: 06: 41.8$ & 0.0812 & 12 & $\mathrm{e}$ & 3 & 18.17 & 0.80 & 0 & 0 & 23.76 \\
\hline 7481 & $13: 05: 38.36$ & $29: 13: 55.0$ & 0.1382 & 51 & $\mathrm{x}$ & 1 & 18.80 & 1.17 & 0 & 0 & 23.95 \\
\hline 7519 & $13: 05: 43.39$ & $29: 25: 41.5$ & 0.0895 & 8 & $\mathrm{e}$ & 4 & 19.62 & 0.76 & 0 & 0 & 23.73 \\
\hline 7566 & $13: 05: 48.89$ & $29: 02: 27.6$ & 2.5800 & 0 & $\mathrm{e}$ & 5 & 19.62 & 0.59 & 0 & 0 & 23.47 \\
\hline 7646 & $13: 06: 00.28$ & $29: 27: 58.2$ & 0.0509 & 8 & $\mathrm{e}$ & 3 & 18.78 & 0.85 & 0 & 0 & 23.88 \\
\hline 7691 & $13: 06: 06.82$ & $29: 09: 37.9$ & 0.1400 & 0 & $\mathrm{e}$ & 3 & 19.07 & 1.03 & 0 & 0 & 23.56 \\
\hline 7766 & $13: 06: 15.19$ & $29: 21: 58.0$ & 0.0246 & 11 & $\mathrm{e}$ & 3 & 18.87 & 0.42 & 0 & 0 & 24.79 \\
\hline 7800 & $13: 06: 21.43$ & $29: 10: 12.0$ & 0.0241 & 39 & $\mathrm{x}$ & 5 & 17.20 & 1.19 & 4 & 1 & 23.68 \\
\hline 7874 & $13: 06: 33.15$ & $29: 11: 02.5$ & 0.0313 & 29 & $\mathrm{c}$ & 4 & 17.15 & 1.05 & 4 & 0 & 23.67 \\
\hline 7886 & $13: 06: 34.23$ & $28: 55: 02.0$ & 0.0611 & 11 & $\mathrm{e}$ & 3 & 19.57 & 0.66 & 0 & 0 & 24.33 \\
\hline 7915 & $13: 06: 37.80$ & $29: 28: 30.6$ & 0.0842 & 11 & $\mathrm{e}$ & 3 & 19.08 & 1.09 & 0 & 0 & 23.84 \\
\hline 7924 & $13: 06: 39.08$ & $29: 03: 48.4$ & 0.2776 & 6 & $\mathrm{e}$ & 3 & 19.99 & 0.84 & 0 & 0 & 24.18 \\
\hline
\end{tabular}


TABLE 3-Continued

\begin{tabular}{|c|c|c|c|c|c|c|c|c|c|c|c|}
\hline $\begin{array}{r}\text { NSER } \\
\text { (1) }\end{array}$ & $\begin{array}{l}\text { R.A. } \\
2000.0 \\
(2)\end{array}$ & $\begin{array}{l}\text { Dec. } \\
2000.0 \\
(3)\end{array}$ & $\begin{array}{c}\mathrm{z} \\
(4)\end{array}$ & $\begin{array}{c} \pm \\
\mathrm{kms}^{-1} \\
(5)\end{array}$ & $\begin{array}{l}\mathrm{t} \\
(6)\end{array}$ & $\begin{array}{l}\mathrm{q} \\
(7)\end{array}$ & $\begin{array}{l}\mathrm{b}_{J} \\
(8)\end{array}$ & $\begin{array}{c}\mathrm{b}_{J}-\mathrm{r}_{F} \\
(9)\end{array}$ & $\begin{array}{c}\operatorname{sat}\left(\mathrm{b}_{J}\right) \\
\% \\
(10)\end{array}$ & $\begin{array}{c}\operatorname{sat}\left(\mathrm{r}_{F}\right) \\
\% \\
(11)\end{array}$ & $\begin{array}{c}\mathrm{SB} \\
\mathrm{b}_{J} \operatorname{arcsec}^{-2} \\
(12)\end{array}$ \\
\hline 7949 & $13: 06: 41.88$ & $28: 54: 24.0$ & 0.0255 & 38 & $\mathrm{x}$ & 5 & 17.96 & 1.19 & 3 & 1 & 23.47 \\
\hline 7957 & $13: 06: 43.12$ & $29: 10: 52.6$ & 0.1378 & 52 & $\mathrm{x}$ & 3 & 19.01 & 1.19 & 0 & 0 & 24.15 \\
\hline 7960 & $13: 06: 43.51$ & $29: 05: 27.4$ & 0.1462 & 15 & e & 3 & 19.65 & 0.94 & 0 & 0 & 23.78 \\
\hline 7994 & $13: 06: 46.88$ & $29: 07: 49.9$ & 0.0268 & 52 & $\mathrm{x}$ & 4 & 17.75 & 1.09 & 0 & 0 & 24.23 \\
\hline 8025 & $13: 06: 50.33$ & $29: 10: 57.2$ & 0.1381 & 9 & $\mathrm{e}$ & 2 & 18.82 & 1.06 & 0 & 0 & 24.09 \\
\hline 8054 & $13: 06: 53.93$ & $29: 14: 28.6$ & 0.1373 & 13 & $\mathrm{e}$ & 3 & 19.22 & 0.93 & 0 & 0 & 23.72 \\
\hline 8059 & $13: 06: 54.57$ & $28: 59: 51.7$ & 0.1856 & 57 & $\mathrm{x}$ & 4 & 19.03 & 1.30 & 0 & 0 & 24.09 \\
\hline 8081 & $13: 06: 57.17$ & $29: 29: 55.2$ & 0.1818 & 9 & e & 3 & 19.54 & 1.03 & 0 & 0 & 23.92 \\
\hline 8087 & $13: 06: 57.86$ & 29:13:31.9 & 0.1675 & 7 & e & 3 & 20.37 & 0.84 & 0 & 0 & 24.80 \\
\hline 8094 & $13: 06: 58.90$ & $29: 09: 56.2$ & 0.0607 & 8 & e & 4 & 18.78 & 0.59 & 0 & 0 & 24.27 \\
\hline 8103 & $13: 07: 00.62$ & $29: 13: 20.2$ & 0.1675 & 5 & $\mathrm{e}$ & 4 & 19.12 & 1.01 & 0 & 0 & 23.62 \\
\hline 8158 & $13: 07: 06.91$ & $29: 29: 39.5$ & 0.1807 & 44 & e & 1 & 18.88 & 1.24 & 0 & 0 & 24.01 \\
\hline 8180 & $13: 07: 10.02$ & $28: 58: 48.9$ & 0.1593 & 45 & $\mathrm{e}$ & 3 & 18.93 & 1.33 & 0 & 0 & 23.98 \\
\hline 8279 & $13: 07: 21.10$ & $28: 59: 44.6$ & 0.1896 & 7 & $\mathrm{e}$ & 4 & 19.41 & 0.65 & 0 & 0 & 23.92 \\
\hline 8410 & $13: 07: 39.34$ & 29:01:05.1 & 0.1236 & 64 & $\mathrm{x}$ & 3 & 18.53 & 1.08 & 0 & 0 & 24.37 \\
\hline 8506 & $13: 07: 53.53$ & $28: 58: 51.2$ & 0.2163 & 7 & $\mathrm{e}$ & 4 & 19.71 & 0.90 & 0 & 0 & 23.65 \\
\hline 9611 & $13: 10: 26.85$ & $29: 11: 41.9$ & 0.2364 & 158 & $\mathrm{x}$ & 1 & 20.23 & 1.38 & 0 & 0 & 24.76 \\
\hline 9707 & $13: 10: 40.43$ & $29: 19: 52.9$ & 0.2434 & 81 & $\mathrm{x}$ & 2 & 20.33 & 1.67 & 0 & 0 & 24.32 \\
\hline 9805 & $13: 10: 55.43$ & $29: 07: 46.2$ & 0.2312 & 110 & $\mathrm{x}$ & 2 & 19.04 & 0.98 & 0 & 0 & 24.21 \\
\hline 9814 & $13: 10: 57.01$ & $29: 14: 00.3$ & 0.2722 & 86 & $\mathrm{x}$ & 3 & 19.73 & 1.37 & 0 & 0 & 24.54 \\
\hline 9822 & $13: 10: 58.49$ & $29: 12: 18.2$ & 0.3038 & 34 & $\mathrm{e}$ & 1 & 20.58 & 0.99 & 0 & 0 & 24.12 \\
\hline 9840 & $13: 11: 01.06$ & $28: 54: 43.1$ & 0.1973 & 32 & $\mathrm{e}$ & 4 & 20.41 & 1.30 & 0 & 0 & 24.68 \\
\hline 9848 & $13: 11: 01.68$ & $29: 34: 41.1$ & 0.0241 & 28 & c & 5 & 16.34 & $\ldots$ & 1 & $\ldots$ & 24.23 \\
\hline 9931 & $13: 11: 12.94$ & $29: 10: 27.5$ & 0.1762 & 26 & $\mathrm{e}$ & 1 & 21.10 & 0.75 & 0 & 0 & 24.98 \\
\hline 10012 & $13: 11: 23.16$ & $29: 22: 14.7$ & 0.2510 & 99 & $\mathrm{x}$ & 1 & 19.95 & 1.45 & 0 & 0 & 24.79 \\
\hline 10075 & $13: 11: 32.12$ & $29: 25: 46.9$ & 0.1868 & 23 & $\mathrm{e}$ & 3 & 20.38 & 1.36 & 0 & 0 & 24.11 \\
\hline 10133 & $13: 11: 39.70$ & $29: 15: 59.7$ & 0.0603 & 28 & c & 3 & 19.97 & 0.40 & 0 & 0 & 24.53 \\
\hline 10160 & $13: 11: 42.32$ & $29: 13: 48.6$ & 0.0843 & 34 & $\mathrm{e}$ & 1 & 20.35 & 0.17 & 0 & 0 & 24.75 \\
\hline 10296 & $13: 12: 01.22$ & $28: 57: 52.9$ & 0.3971 & 27 & $\mathrm{e}$ & 4 & 20.43 & 1.34 & 0 & 0 & 24.25 \\
\hline 10444 & $13: 12: 21.38$ & $29: 21: 38.9$ & 0.1734 & 60 & $\mathrm{x}$ & 5 & 18.66 & 1.50 & 0 & 0 & 24.02 \\
\hline 10447 & $13: 12: 21.69$ & $29: 19: 20.9$ & 0.1240 & 126 & $\mathrm{x}$ & 4 & 19.91 & 1.27 & 0 & 0 & 24.08 \\
\hline 10493 & $13: 12: 27.51$ & $29: 06: 49.9$ & 0.1219 & 25 & $\mathrm{e}$ & 3 & 20.71 & 0.94 & 0 & 0 & 24.17 \\
\hline 10519 & $13: 12: 31.25$ & $29: 15: 32.4$ & 0.1975 & 100 & $\mathrm{x}$ & 4 & 19.87 & 1.40 & 0 & 0 & 24.29 \\
\hline 10543 & $13: 12: 33.97$ & $29: 17: 54.7$ & 0.1224 & 40 & $\mathrm{x}$ & 5 & 18.93 & 1.08 & 0 & 0 & 23.93 \\
\hline 10564 & $13: 12: 36.32$ & $29: 09: 23.7$ & 0.0721 & 32 & $\mathrm{e}$ & 3 & 19.27 & 0.77 & 0 & 0 & 24.15 \\
\hline 10651 & $13: 12: 47.04$ & $29: 13: 15.6$ & 0.2395 & 119 & $\mathrm{x}$ & 4 & 19.91 & 1.15 & 0 & 0 & 24.35 \\
\hline 10667 & $13: 12: 48.71$ & $29: 23: 18.7$ & 0.1717 & 124 & $\mathrm{x}$ & 3 & 20.54 & 1.34 & 0 & 0 & 24.28 \\
\hline 10669 & $13: 12: 48.93$ & $29: 22: 23.8$ & 0.2634 & 33 & $\mathrm{e}$ & 4 & 19.71 & 1.08 & 0 & 0 & 24.42 \\
\hline 10677 & $13: 12: 49.70$ & $29: 18: 15.2$ & 0.2407 & 130 & $\mathrm{x}$ & 4 & 19.51 & 1.65 & 0 & 0 & 24.23 \\
\hline 10750 & $13: 12: 59.25$ & $29: 13: 17.6$ & 0.2360 & 118 & $\mathrm{x}$ & 4 & 20.15 & 1.57 & 0 & 0 & 24.23 \\
\hline
\end{tabular}

Note.-Col. (1) serial number in catalog. Col. (2) right ascension. Col (3) declination. Col. (4) heliocentric redshift. Col. (5) internal error of redshift. Col. (6) redshift type: cross-correlation (x), emission (e) and combined emission and cross-correlation (c). Col. (7) redshift quality indicating number of features identified in spectrum. Col. (8) magnitude. Col. (9) color. Cols. (10) and (11) percentage estimate of the number of pixels close to saturation for the $\mathrm{b}_{J}$ and $\mathrm{r}_{F}$ catalogs respectively. Col. (12) estimate of the mean surface brightness for the $b_{J}$ plate catalog. 


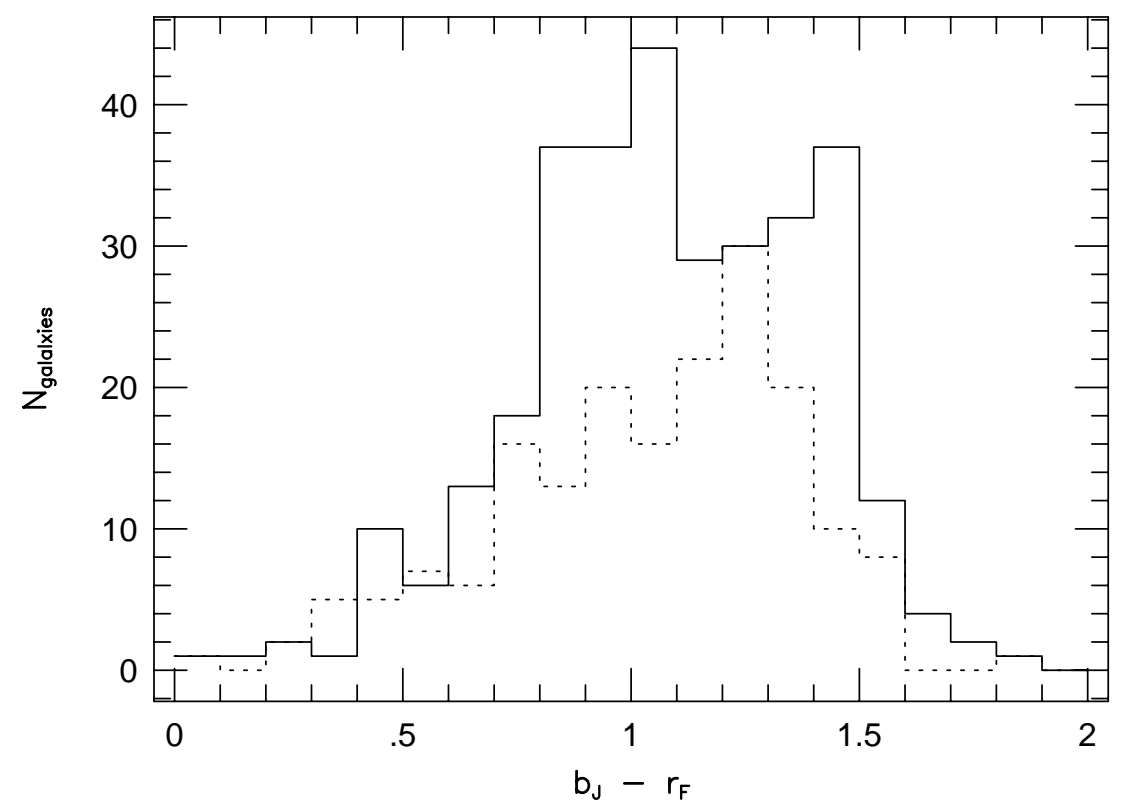

